\title{
Intercomparison of general circulation models for hot extrasolar planets
}

Article

Accepted Version

Polichtchouk, I., Cho, J. Y.-K., Watkins, C., Thrastarson, H. T., Umurhan, O. M. and de la Torre Juárez, M. (2014) Intercomparison of general circulation models for hot extrasolar planets. Icarus, 229. pp. 355-377. ISSN 0019-1035 doi: https://doi.org/10.1016/j.icarus.2013.11.027 Available at https://centaur.reading.ac.uk/38492/

It is advisable to refer to the publisher's version if you intend to cite from the work. See Guidance on citing.

To link to this article DOI: http://dx.doi.org/10.1016/j.icarus.2013.11.027

Publisher: Elsevier

All outputs in CentAUR are protected by Intellectual Property Rights law, including copyright law. Copyright and IPR is retained by the creators or other copyright holders. Terms and conditions for use of this material are defined in the End User Agreement.

\section{www.reading.ac.uk/centaur}

\section{CentAUR}

Central Archive at the University of Reading 
Reading's research outputs online 


\title{
Intercomparison of General Circulation Models for Hot Extrasolar Planets
}

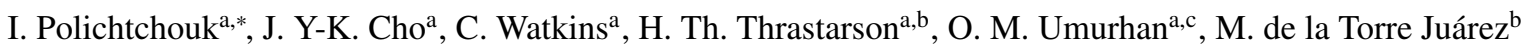 \\ ${ }^{a}$ School of Physics and Astronomy, Queen Mary University of London, London E1 4NS, UK \\ ${ }^{b}$ Jet Propulsion Laboratory, California Institute of Technology, Pasadena, CA 91109, USA \\ ${ }^{c}$ School of Natural Sciences, University of California, Merced, CA 95343, USA
}

\begin{abstract}
We compare five general circulation models (GCMs) which have been recently used to study hot extrasolar planet atmospheres (BOB, CAM, IGCM, MITgcm, and PEQMOD), under three test cases useful for assessing model convergence and accuracy. Such a broad, detailed intercomparison has not been performed thus far for extrasolar planets study. The models considered all solve the traditional primitive equations, but employ different numerical algorithms or grids (e.g., pseudospectral and finite volume, with the latter separately in longitude-latitude and 'cubed-sphere' grids). The test cases are chosen to cleanly address specific aspects of the behaviors typically reported in hot extrasolar planet simulations: 1) steady-state, 2) nonlinearly evolving baroclinic wave, and 3) response to fast timescale thermal relaxation. When initialized with a steady jet, all models maintain the steadiness, as they should-except MITgcm in cubed-sphere grid. A very good agreement is obtained for a baroclinic wave evolving from an initial instability in pseudospectral models (only). However, exact numerical convergence is still not achieved across the pseudospectral models: amplitudes and phases are observably different. When subject to a typical 'hot-Jupiter'-like forcing, all five models show quantitatively different behavior - although qualitatively similar, time-variable, quadrupole-dominated flows are produced. Hence, as have been advocated in several past studies, specific quantitative predictions (such as the location of large vortices and hot regions) by GCMs should be viewed with caution. Overall, in the tests considered here, pseudospectral models in pressure coordinate (PEBOB and PEQMOD) perform the best and MITgcm in cubed-sphere grid performs the worst.
\end{abstract}

Keywords: Extra-solar planets; Atmospheres, dynamics; Meteorology.

\section{Introduction}

Carefully testing general circulation models (GCMs) of extrasolar planets is important for understanding the physical properties of the atmospheres and for attaining confidence in the complex models themselves. Intercomparison of full GCMs, as well as benchmarking of dynamical cores and testbed models against 'standard solutions', are common in Earth studies (e.g., Held and Suarez, 1994; Boer and Denis, 1997; Polvani et al., 2004; Jablonowski and Williamson, 2006). Intercomparisons are also becoming more common for circulation models of other Solar System planets (e.g., Lebonnois et al., 2013). However, similar intercomparisons have not been performed for models of hot extrasolar planets. Given that the conditions of many extrasolar planets are markedly different than the Earthand much more exacting on the circulation models-it is useful to subject the models to tests which are appropriate for extrasolar conditions (e.g., Thrastarson and Cho, 2011).

Thus far, only Rauscher and Menou (2010) and Heng et al. (2011) have explicitly attempted to intercompare simulations of hot extrasolar planets performed with different GCMs. The former study attempts to compare their results using the Intermediate General Circulation Model (Blackburn, 1985) with those reported in Cooper and Showman (2005) using the ARIES/GEOS

\footnotetext{
${ }^{*}$ Corresponding author

Email address: i.polichtchouk@qmul .ac.uk (I. Polichtchouk)
}

model (Suarez and Takacs, 1995). However, while qualitatively similar features were observed, the comparison was somewhat inconclusive because the model setup was not identical. In their studies using the Community Atmosphere Model (Collins et al., 2004), Thrastarson and Cho $(2010,2011)$ have shown sensitivity to initial condition, as well as thermal relaxation and explicit numerical dissipation specifications. A clearer comparison than in Rauscher and Menou (2010) has been presented in Heng et al. (2011). In the latter study, time-mean zonally-averaged (i.e., longitudinally-averaged) fields are presented from simulations with the Flexible Modeling System (Anderson et al., 2004), using two different types of numerical algorithm (pseudospectral and finite volume). However, while zonal and temporal averaging is somewhat justifiable for rapidly rotating planets, the procedure is less useful for the more slowly rotating planets, such as those considered in the study: the averaging can destroy dynamically-significant flow structures, as well as conceal subtle numerical and coding errors. ${ }^{1}$

In addition to the setup not being same or systematic across different models, the inconclusiveness of the past comparisons and the general variability of the model results stem from the

${ }^{1}$ During the preparation of this manuscript another study, by Bending et al. (2013) appeared that compares their results with those of Menou and Rauscher (2009). The authors of the new study report that they are not able to reproduce precisely the results of the older study, although both studies use the same dynamical core (Section 2.1). 
fact that the models employ different numerical algorithms, grids, and coordinates to solve the governing equations-as we shall show in this work. Moreover, the numerical parameters of the model calculations are often not described explicitly in the literature, or even in the technical documentations of the models themselves. Thus, it has been difficult to ascertain which differences between model outputs are due to the model and which are due to the setup. Here, we perform a careful comparison of five GCMs recently used to study hot extrasolar planet atmospheres. The GCMs are: $\mathrm{BOB}^{2}, \mathrm{CAM}^{3}, \mathrm{IGCM}^{4}, \mathrm{MITgcm}^{5}$, and PEQMOD ${ }^{6}$. They have been used, for example, in the following extrasolar planet circulation studies: BOB (Beaulieu et al., 2011; Polichtchouk and Cho, 2012), CAM (Thrastarson and Cho, 2010, 2011), IGCM (Menou and Rauscher, 2009; Rauscher and Menou, 2010), MITgcm (Showman et al., 2009; Lewis et al., 2010), and PEQMOD (Cho and Polichtchouk, in prep.).

The five GCMs are submitted to three tests which are useful for assessing model convergence and accuracy. The tests are chosen to specifically address three features that have been typically reported in hot extrasolar planet atmospheric flow simulations: 1) steady flow, 2) nonlinear baroclinic wave, and 3) response to a fast timescale thermal relaxation. We stress that, in addition to their good range and relevance, the tests are purposely chosen with reproducibility of the results in mind: the tests are not difficult to set up and full descriptions of the test cases (as well as the GCMs tested) are provided, along with all of the model parameter values used in the simulations (see Appendix) — as per our usual practice. We are also happy to share all source codes and input files/parameters used in this study. Note that the emphasis in this work is on models tested in their 'default configuration' (i.e., essentially as they are unpacked), modulo minor modifications to facilitate equatable (as well as equitable $)^{7}$ comparisons.

The overall plan of the paper is as follows. In Section 2, we review the governing equations solved by the five GCMs and describe the discretization and dissipation schemes used in the models. In Section 3, the three test cases are carefully described and the results from the tests are presented in turn. Both inter-model and intra-model comparisons are presented in detail, where the former comparison refers to 'between different models' and the latter comparison refers to 'within a single model'. The aim of this section-indeed, of this entire paperis to permit one to go beyond broad-brush comparisons based on strongly dissipated/constrained or averaged fields. In Section 4, summary and conclusions are given, along with some discussion of implications of this work.

\footnotetext{
${ }^{2}$ Built on Beowolf (Scott et al., 2003)

${ }^{3}$ Community Atmosphere Model - version 3.0 (Collins et al., 2004)

${ }^{4}$ Intermediate General Circulation Model (Blackburn, 1985)

${ }^{5}$ MIT general circulation model - checkpoint64d (Adcroft et al., 2012)

${ }^{6}$ Primitive Equations Model (Saravanan, 1992)

${ }^{7}$ Equitable refers to 'impartial' or 'fair', and equatable refers to 'equivalent' or 'comparable'.
}

\section{Dynamical Cores and Test Cases}

\subsection{Dynamical Cores}

The GCMs - or, more precisely, their 'dynamical cores' discussed in this work all solve the hydrostatic primitive equations for the 'dry' atmosphere. The dynamical core is essentially that part of the GCM which remains when all the sophisticated physical parameterizations (e.g., convection, radiation, wave-drag, etc.) have been stripped away: it is the engine of the GCM. In this paper, we refer to 'GCMs' and 'dynamical cores' interchangeably, as the distinction is not particularly important here. None of the sophisticated physical parameterizations are used in any of the models for the comparisons: only a crude heating/cooling scheme is used in one of the test cases. In general, it is prudent to test and characterize the core before moving onto the full GCM. ${ }^{8}$.

The equations solved govern the large-scale dynamics of planetary atmospheres (e.g., Holton (1992); see also Cho (2008) for some discussions relevant to the current work). Given that the GCMs tested solve the equations in different vertical coordinate systems (e.g., pressure, sigma, eta-see below), we first present and discuss the equations in the generalized vertical coordinate, $s$. In the $s$-coordinate, the hydrostatic primitive equations read

$$
\begin{aligned}
\frac{\mathrm{D} \mathbf{v}}{\mathrm{D} t} & =-\frac{1}{\rho} \boldsymbol{\nabla}_{s} p-g \boldsymbol{\nabla}_{s} z-f \mathbf{k} \times \mathbf{v}+\mathcal{F}_{\mathbf{v}}+\mathcal{D}_{\mathbf{v}} \\
\frac{\mathrm{D} \theta}{\mathrm{D} t} & =\frac{\theta}{c_{p} T} \dot{q}_{\mathrm{net}}+\mathcal{D}_{\theta} \\
\frac{\partial p}{\partial s} & =-\rho g \frac{\partial z}{\partial s} \\
0 & =\frac{\partial}{\partial s}\left(\frac{\partial p}{\partial t}\right)_{s}+\nabla_{s} \cdot\left(\mathbf{v} \frac{\partial p}{\partial s}\right)+\frac{\partial}{\partial s}\left(\dot{s} \frac{\partial p}{\partial s}\right),
\end{aligned}
$$

where

$$
\frac{\mathrm{D}}{\mathrm{D} t} \equiv\left(\frac{\partial}{\partial t}\right)_{s}+\mathbf{v} \cdot \boldsymbol{\nabla}_{s}+\dot{s} \frac{\partial}{\partial s} .
$$

Here, $\mathbf{v}(\mathbf{x}, s, t)=(u, v)$ is the (zonal, meridional) velocity in the frame rotating with $\boldsymbol{\Omega}$, the planetary rotation vector, and $\mathbf{x} \in \mathbb{R}^{2} ; \dot{s} \equiv \mathrm{D} s / \mathrm{D} t$ is the generalized vertical velocity; $z=z(\mathbf{x}, s, t)$ is the physical height, directed locally upward (in the direction of the unit vector $\mathbf{k}) ; \boldsymbol{\nabla}_{s}$ is the two-dimensional (2D) gradient operator, operating along constant surfaces of $s=s(\mathbf{x}, z, t) ; \rho(\mathbf{x}, s, t)$ is the density; $p(\mathbf{x}, s, t)$ is the pressure; $f=2 \Omega \sin \phi=2 \boldsymbol{\Omega} \cdot \mathbf{k}$ is the Coriolis parameter, where $\phi$ is the latitude; $\mathcal{F}_{\mathbf{v}}(\mathbf{x}, s, t)$ represents momentum sources; $\mathcal{D}_{\mathbf{v}}(\mathbf{x}, s, t)$ and $\mathcal{D}_{\theta}(\mathbf{x}, s, t)$ represent momentum and potential temperature sinks, respectively; $g$ is the gravitational acceleration, assumed to be constant and to include the centrifugal acceleration contribution; $\theta(\mathbf{x}, s, t)=T\left(p_{\mathrm{r}} / p\right)^{\kappa}$ is the potential temperature, where $T(\mathbf{x}, s, t)$ is the temperature, $p_{\mathrm{r}}$ is a constant reference pressure, and $\kappa=\mathcal{R} / c_{p}$, with $\mathcal{R}$ the specific gas constant and $c_{p}$ the constant specific heat at constant pressure; and, $\dot{q}_{\text {net }}(\mathbf{x}, s, t)$ is the net diabatic heating rate (i.e., heating minus cooling).

\footnotetext{
${ }^{8}$ Note that, in comparisons of full GCMs for the Earth, model differences generally increase when physics parameterizations are included (e.g., Blackburn et al. (2013))
} 
The set of equations, (1a)-(1d), is closed with the ideal gas equation of state, $p=\rho \mathcal{R} T$. The equation set is also supplemented with the boundary conditions,

$$
\begin{aligned}
& \dot{s}=0 \quad \text { at } \quad s=s_{\mathrm{T}} \\
& \dot{s}=\frac{\partial s_{\mathrm{B}}}{\partial t}+\mathbf{v}_{\mathrm{B}} \cdot \boldsymbol{\nabla} s_{\mathrm{B}} \quad \text { at } \quad s=s_{\mathrm{B}} .
\end{aligned}
$$

Here, $s_{\mathrm{T}}$ is the boundary surface at the top; $s_{\mathrm{B}}$ is the boundary surface at the bottom, at a fixed altitude above the reference height $(z=0)$; and, $\mathbf{v}_{\mathrm{B}}$ is horizontal velocity at the bottom surface. Boundary conditions (2) imply no mass transport through the upper and lower boundary surfaces. Note, if the lower boundary coincides with a constant $s$-surface (i.e., $\left.s_{\mathrm{B}} \neq s_{\mathrm{B}}(\mathbf{x}, t)\right)$, then the boundary condition (2b) simply reduces to

$$
\dot{s}=0 \quad \text { at } s=s_{\mathrm{B}} .
$$

While letting $s \rightarrow z$ might be an intuitive choice for a vertical coordinate, it is common in GCMs to use pressure or other, pressure-based, coordinates: for example, $s \rightarrow p, s \rightarrow \sigma(p)$, or $s \rightarrow \eta(p)$. In the $p$-coordinate, the continuity equation (1d) takes on a simple diagnostic form. However, the coordinate system poses a computational disadvantage when modelling a planet with a solid surface, if topography is present. In this case, the boundary condition (2b) becomes difficult to handle. The $\sigma$-coordinate or the $\eta$-coordinate circumvents this problem because the planet's surface does not intersect a vertical coordinate surface in either coordinate systems.

All the cores tested solve the equations in the Eulerian framework. Hence, for all of them, there is an associated grid for the computational domain-e.g., longitude-latitude (LL) and cubed-sphere (CS) grids for the MITgcm core and the Gaussian grid for the remaining four cores. The numerical integrations are directly performed on the LL and CS grids in MITgcm, while only the nonlinear products are evaluated and initial conditions are specified on the Gaussian grid in the pseudospectral cores. More details on the cores are provided below, beginning with the pseudospectral ones. All cores use common values for the planetary parameters, which are listed in Table 1: the values characteristic of the planet HD209458b are used.

\subsubsection{Pseudospectral Cores}

BOB, CAM, IGCM, and PEQMOD cores use the highlyaccurate pseudospectral algorithm (Orszag, 1970; Eliassen et al., 1970; Canuto et al., 1988) for the horizontal direction. Equations (1) in the vorticity-divergence form are transformed with triangular truncation (i.e., $M=N$, where $M$ is the maximum zonal wavenumber and $N$ is the maximum total wavenumber retained in the spherical harmonic expansion). ${ }^{9}$ Given $M$ and $N$, all the nonlinear products in the full set of equations (1) are first evaluated in physical space on a Gaussian grid with enough points, in principle, to eliminate aliasing errors and then

${ }^{9}$ IGCM uses the 'jagged triangular truncation', in which $n \leq M-1$ for variables even about the equator and $n \leq M$ for variables odd about the equator. Here, $n$ is the total wavenumber.
Table 1: Parameter values for the hot extrasolar planet HD209458b. Here, $g$ is surface gravity; $R_{p}$ is equatorial radius; $\Omega$ is rotation rate; $\mathcal{R}$ is gas constant; $c_{p}$ is specific heat at constant pressure; and, $T_{\text {eq }}$ is equilibrium temperature.

\begin{tabular}{lll}
\hline Parameter & Value & Units \\
\hline$g$ & 9.8 & $\mathrm{~m} \mathrm{~s}^{-2}$ \\
$R_{p}$ & $10^{8}$ & $\mathrm{~m}$ \\
$\Omega$ & $2.1 \times 10^{-5}$ & $\mathrm{~s}^{-1}$ \\
$\mathcal{R}$ & $3.5 \times 10^{3}$ & $\mathrm{~J} \mathrm{~kg}^{-1} \mathrm{~K}^{-1}$ \\
$c_{p}$ & $1.23 \times 10^{4}$ & $\mathrm{~J} \mathrm{~kg}^{-1} \mathrm{~K}^{-1}$ \\
\hline
\end{tabular}

transformed to spectral space. The linear terms are directly transformed.

For the vertical direction, a standard second-order finite difference scheme is used. In this direction, the grid is typically equally spaced in $p$-coordinates or $\sigma$-coordinates, as are all the simulations in this work (see below). Note that all the cores tested in this work use the Lorenz grid (Lorenz, 1960), in which the vertical velocity is defined at the boundary of the layers and the prognostic variables (e.g., vorticity, divergence, and temperature/potential temperature) are defined at the centers of the layers. The grid allows boundary conditions of no flux at the top and bottom of the domain to be easily fulfilled. However, a spurious computational mode is admitted, arising from an extra degree of freedom introduced in the potential temperature (Arakawa and Moorthi, 1988). We have observed that this can lead to small-amplitude oscillations in the temperature field on the timescale of a timestep.

As for the time integration, the above four cores use a semiimplicit leap-frog scheme. A small Robert-Asselin time filter coefficient $\epsilon$ (Robert, 1966; Asselin, 1972) is applied at every timestep in each layer to filter the computational mode arising from using the second-order time-marching scheme (see, e.g., Thrastarson and Cho, 2010). To integrate the equations over long simulation durations, explicit dissipation is applied to the prognostic variables so that artificial accumulation of energy at small scales is prevented (e.g., Cho and Polvani, 1996; Thrastarson and Cho, 2011). The dissipations, $\mathcal{D}_{\mathbf{v}, \theta}$ in equations (1), are in the form of a 'hyperdissipation' operator (see equation (5) below).

BOB and PEQMOD solve equations (1) in the $p$-coordinate:

$$
\begin{aligned}
& \frac{\partial \zeta}{\partial t}=\mathbf{k} \cdot \boldsymbol{\nabla} \times \mathbf{n}_{\mathrm{p}}+\mathcal{D}_{\zeta} \\
& \frac{\partial \delta}{\partial t}=\boldsymbol{\nabla} \cdot \mathbf{n}_{\mathrm{p}}-\nabla^{2}(E+\Phi)+\mathcal{D}_{\delta} \\
& \frac{\partial \Phi}{\partial \xi}=-c_{p} \theta \\
& \frac{\partial \omega}{\partial p}=-\delta \\
& \frac{\partial \theta}{\partial t}=-\boldsymbol{\nabla} \cdot(\theta \mathbf{v})-\frac{\partial(\omega \theta)}{\partial p}+\frac{\theta}{c_{p} T} \dot{q}_{\mathrm{net}}+\mathcal{D}_{\theta},
\end{aligned}
$$


where $\zeta=\mathbf{k} \cdot(\boldsymbol{\nabla} \times \mathbf{v})$ is the relative vorticity;

$$
\mathbf{n}_{\mathrm{p}}=-(\zeta+f) \mathbf{k} \times \mathbf{v}-\delta \mathbf{v}-\frac{\partial(\omega \mathbf{v})}{\partial p}
$$

$\delta=\boldsymbol{\nabla} \cdot \mathbf{v}$ is the divergence; $E=(\mathbf{v} \cdot \mathbf{v}) / 2$ is the specific kinetic energy; $\Phi=g z$ is the specific geopotential above the planetary radius $R_{p} ; \omega=\mathrm{D} p / \mathrm{D} t$ is the vertical velocity, where

$$
\frac{\mathrm{D}}{\mathrm{D} t} \equiv \frac{\partial}{\partial t}+\mathbf{v} \cdot \boldsymbol{\nabla}+\omega \frac{\partial}{\partial p}
$$

is the material derivative with $\boldsymbol{\nabla}$ operating along constant surfaces of $p$; and, the diffusion terms $\mathcal{D}_{\chi}$, where $\chi=\{\zeta, \delta, \theta\}$, are given by (e.g., Cho and Polvani, 1996):

$$
\mathcal{D}_{\chi}=v_{2 \mathfrak{p}}\left[(-1)^{\mathfrak{p}+1} \nabla^{2 \mathfrak{p}}+C\right] \chi,
$$

where $\mathfrak{p}$ (different from $p$, the pressure) is the order of the hyperdissipation operator; $v_{2 \mathfrak{p}}$ is the hyperdissipation coefficient; and, $C=\left(2 / R_{p}^{2}\right)^{\mathfrak{p}}$ is a correction term added to the vorticity and divergence equations to prevent damping of uniform rotations for angular momentum conservation. Note that, in the baroclinic wave test case, $\mathfrak{p}=1$, leading to the normal Laplacian dissipation. In the diabatic test case, $\mathfrak{p}=2$, leading to the more scale-selective 'superdissipation'.

BOB and PEQMOD have an additional constraint of no vertically integrated divergence over the whole atmosphere. This constraint excludes the divergent 'shallow-water mode', which has a barotropic vertical structure, and increases the computationally stability of the models. With this additional constraint, the boundary conditions (2) become

$$
\omega=0 \quad \text { at } \quad p=0, p_{\mathrm{B}}
$$

and the lower boundary always coincides with a constant $p$ surface. The above boundary conditions entail zero flux of any quantity through the upper and lower pressure surfaces.

As already discussed, in the $p$-coordinate continuity equation (1c) simplifies to a simple diagnostic equation (4d). Thus, with the boundary conditions (6), BOB and PEQMOD actually only integrate three equations - that is, only three variables are prognostic. The vertical discretization scheme in BOB and PEQMOD preserves the global conservation properties of absolute angular momentum, potential temperature and total energy in the absence of forcing/dissipation. Note, unlike BOB, PEQMOD implements a slightly non-standard Gaussian transform grid, for which the equatorial point is constrained to be one of the Gaussian points.

IGCM is formulated in the $\sigma$-coordinate: $\sigma=p / p_{\mathrm{s}}$, where $p_{\mathrm{s}}$ is the surface pressure. This coordinate system is specifically designed to 'follow the terrain' at the bottom. CAM is formulated in a more general, hybrid terrain-following vertical coordinate, $\eta$ : this coordinate system allows the upper part of the model atmosphere to be represented by $p$-coordinates and the lower part of the model atmosphere by $\sigma$-coordinates. To ensure equitable model inter-comparison, we have set $\eta$ so that $\eta=\sigma$ throughout the vertical domain in CAM. In the $\sigma-$ coordinate the vorticity-divergence form of the primitive equations read:

$$
\begin{aligned}
\frac{\partial \zeta}{\partial t} & =\mathbf{k} \cdot \boldsymbol{\nabla} \times \mathbf{n}_{\sigma}+\mathcal{D}_{\zeta} \\
\frac{\partial \delta}{\partial t} & =\boldsymbol{\nabla} \cdot \mathbf{n}_{\sigma}-\nabla^{2}(E+\Phi)+\mathcal{D}_{\delta} \\
\frac{\partial \Phi}{\partial \sigma} & =-\frac{\mathcal{R} T}{p} \frac{\partial p}{\partial \sigma} \\
\frac{\partial p_{\mathrm{s}}}{\partial t} & =\int_{1}^{0} \boldsymbol{\nabla} \cdot\left(p_{\mathrm{s}} \mathbf{v}\right) \mathrm{d} \sigma^{\prime} \\
\frac{\partial T}{\partial t} & =-\mathbf{v} \cdot \boldsymbol{\nabla} T-\dot{\sigma} \frac{\partial T}{\partial \sigma}-\frac{\kappa T \omega}{p}+\frac{\dot{q}}{c_{p}}+\mathcal{D}_{\mathcal{T}}
\end{aligned}
$$

where

$$
\mathbf{n}_{\sigma}=-(\zeta+f) \mathbf{k} \times \mathbf{v}-\dot{\sigma} \frac{\partial \mathbf{v}}{\partial \sigma}-\frac{\mathcal{R} T}{p} \boldsymbol{\nabla} p
$$

with $\dot{\sigma} \equiv \mathrm{D} \sigma / \mathrm{D} t$ and $\mathrm{D} / \mathrm{D} t \equiv \partial / \partial t+\mathbf{v} \cdot \boldsymbol{\nabla}+\dot{\sigma} \partial / \partial \sigma$, the material derivative; $\mathcal{D}_{\chi}$, where $\chi=\{\zeta, \delta, T\}$, are given by equation (5); and, $\boldsymbol{\nabla}$ here acts on the constant $\sigma$ surfaces. Note that these set of equations are slightly different than equations (4).

For example, the continuity equation ( $7 \mathrm{~d}$ ) comes in the form of a prognostic equation for surface pressure $p_{\mathrm{s}}$ and is obtained by integrating the continuity equation (1c) from the bottom $(\sigma=1)$ to the top $(\sigma=0)$ surfaces and using the boundary conditions,

$$
\dot{\sigma}=0 \quad \text { at } \quad \sigma=0,1 .
$$

The pressure vertical velocity, $\omega=\mathrm{D} p / \mathrm{D} t$, is computed diagnostically from the definition:

$$
\omega=\sigma \mathbf{v} \cdot \boldsymbol{\nabla} p_{\mathrm{s}}-\int_{0}^{\sigma} \boldsymbol{\nabla} \cdot\left(p_{\mathrm{s}} \mathbf{v}\right) \mathrm{d} \sigma^{\prime} .
$$

Note, IGCM and CAM employ a vertical discretization scheme described by Simmons and Burridge (1981). This vertical finite difference scheme explicitly conserves mass, total energy and angular momentum. ${ }^{10}$

\subsubsection{Finite Volume Core}

The MITgcm core is widely employed by the Earth's atmospheric and oceanic communities. It is highly configurable and is also used in modeling flows on and in Solar System planets (e.g., Kaspi, 2009). MITgcm supports both the traditional hydrostatic and non-hydrostatic formulation of the primitive equations. The model, in its traditional formulation, has been applied in extrasolar planet circulation studies (e.g., Showman et al., 2009; Lewis et al., 2010). In this work, the traditional hydrostatic formulation is used to ensure equatable comparison.

The primitive equations (1) in equally-spaced $p$-coordinate, in spherical geometry, are solved on a staggered Arakawa Cgrid (Arakawa and Lamb, 1977) with a second-order finite volume spatial discretization method (e.g., Durran, 1999) in the LL

\footnotetext{
${ }^{10}$ However, the issue of 'hard-wiring' in select conservation laws in a numerical scheme is a matter of current debate. For example, an adequately resolved calculation arguably does not require a scheme that explicitly enforces global energy conservation, which can sometimes lead to unphysical stabilization and erroneous results.
} 
grid and an enstrophy-conserving ${ }^{11}$ scheme (Sadourny, 1975) on the CS grid-the default configurations of the MITgcm core for the two grids, respectively. ${ }^{12}$ On the LL grid, the grid size approaches zero near the poles; and, to maintain numerical stability given by the Courant-Friedrichs-Lewy (CFL) condition (e.g., Durran, 1999, and references therein), an infinitesimal timestep size is required. To avoid taking very small timesteps, a fast Fourier transform (FFT) filter, which smooths out the physically-insignificant grid-scale waves in the zonal direction, is applied polewards of $45^{\circ}$ at each timestep. The problem of grid singularity at the poles in the LL grid is overcome by the CS grid, which has nearly uniform grid-spacing. This grid allows longer timesteps to be taken at a comparable resolution and eliminates the need for a zonal filter. However, the CS grid introduces eight special 'corner points' (four in each hemisphere), which lead to an intrinsic wavenumber-4 error in both the northern and southern hemispheres (see Section 3.1).

For the timestepping, a third-order Adams-Bashforth scheme (e.g., Durran, 1999) is used. ${ }^{13}$ The third-order AdamsBashforth scheme is more stable, compared with its secondorder counterpart, and does not require a stabilizing parameter to damp the computational mode. This scheme is superior to the second-order leapfrog scheme used in the pseudospectral cores, especially when the second-order scheme is used in conjunction with the Robert-Asselin filter-as is often the case.

MITgcm supports several dissipation schemes, including harmonic and biharmonic dissipations, as well as the Shapiro filter (Shapiro, 1970). Because ordinary harmonic dissipation is easy to implement in both pseudospectral and finite volume cores, Laplacian dissipation (corresponding to $\mathfrak{p}=1$ in equation (5)), is applied to the thermodynamic and momentum equations to control the grid-scale noise in the baroclinic wave test case. This approach is similar to the one in Polvani et al. (2004) and ensures that all models solve the same equations, modulo the vertical coordinate.

Previous extrasolar planet studies with MITgcm have implemented the Shapiro filter (in CS grid) to control the grid scale oscillations (e.g., Showman et al., 2009; Lewis et al., 2010). Since the purpose of the diabatic test case is to facilitate clear interpretation of outputs from current hot extrasolar planet studies, we apply the Shapiro filter in the third test case. ${ }^{14}$

The Shapiro filter is applied to prognostic variable $\chi$, where $\chi=\{\mathbf{v}, \theta\}$, in the zonal and meridional directions. The discrete

\footnotetext{
${ }^{11}$ Enstrophy is $\frac{1}{2} \zeta^{2}$. It is conserved in 2D Euler equation-a 2D, barotropic form of equations (1) with rigid lid and bottom, in the inviscid limit.

${ }^{12}$ We have found embedded in the code two additional schemes, which are not described in the official documentation, for solving the momentum equation on the CS grid. These schemes are not invoked in the default setting, and results from detailed tests are presented in a follow-up paper (Polichtchouk and Cho, in prep.)

${ }^{13}$ Strictly speaking, this scheme is not 'default' in MITgcm. However, we have also tested the second-order Adams-Bashforth scheme, which is the default, and verified that there is no noticeable difference in the results between the two schemes.

${ }^{14}$ Note, we have also performed the third test case with Laplacian dissipation, as well as with the full range of Shapiro filters, for completeness (see Section 3.3).
}

form of the Shapiro filter in MITgcm is:

$$
\tilde{\chi}_{i, j}=\left[1-\frac{\Delta t}{\tau_{\text {shap }}}\left\{\frac{1}{8}\left(\mathrm{~F}_{\lambda}+\mathrm{F}_{\phi}\right)\right\}^{\mathfrak{n}}\right] \chi_{i, j} .
$$

Here, $\chi_{i, j}$ is an arbitrary variable at the longitude and latitude grid points $i$ and $j$, respectively, and is denoted with an overtilde (i.e., $\widetilde{\chi}_{i, j}$ ) when smoothed; $\mathrm{F}_{\lambda}(\cdot)$ and $\mathrm{F}_{\phi}(\cdot)$ are dimensionless operators operating on $\chi_{i, j}$ such that

$$
\begin{aligned}
& \mathrm{F}_{\lambda}\left(\chi_{i, j}\right)=\chi_{i+1, j}-2 \chi_{i, j}+\chi_{i-1, j}, \\
& \mathrm{~F}_{\phi}\left(\chi_{i, j}\right)=\chi_{i, j+1}-2 \chi_{i, j}+\chi_{i, j-1} .
\end{aligned}
$$

The integer $\mathfrak{n}$ (different from $n$, the total wavenumber) is the power of the Shapiro filter; $\lambda$ is the longitude; $\Delta t$ is the timestep size; and, $\tau_{\text {shap }}$ is a parameter which defines the strength of the filter, given $\Delta t$. In Earth circulation studies, low power (i.e., $\mathfrak{n}=\{2,4,6\})$ Shapiro filters are generally avoided and are replaced either by highly scale-selective FFT filters or by less dissipative, $\mathfrak{n}=\{8,16\}$, Shapiro filters. Higher power filters are chosen to avoid over-dissipating the mid-latitude and tropical waves (e.g., Lauritzen et al., 2011). However, we have found that the strong forcing used in hot extrasolar planet studies generally necessitates the use of a more dissipative $(\mathfrak{n} \leq 6)$ filter, for the model in its default configuration. For example, in the diabatic forcing test case, simulations with MITgcm core at C16 resolution in the CS grid crash for $\mathfrak{n} \geq 8$ Shapiro filters for all values of $\tau_{\text {shap }} \geq \Delta t$, with $\Delta t$ comparable to those used in pseudospectral core simulations at similar resolution. ${ }^{15}$

The form of the primitive equations (1) solved by MITgcm in the $p$-coordinate is as follows:

$$
\begin{aligned}
& \frac{\partial \mathbf{v}}{\partial t}=-(\mathbf{v} \cdot \boldsymbol{\nabla}) \mathbf{v}-\omega \frac{\partial \mathbf{v}}{\partial p}-\nabla \Phi-f \mathbf{k} \times \mathbf{v}+\mathcal{D}_{\mathbf{v}} \\
& \frac{\partial \Phi}{\partial p}=-\alpha \\
& \boldsymbol{\nabla} \cdot \mathbf{v}=-\frac{\partial \omega}{\partial p} \\
& \frac{\partial \theta}{\partial t}=-\mathbf{v} \cdot \boldsymbol{\nabla} \theta-\omega \frac{\partial \theta}{\partial p}+\frac{\theta}{c_{p} T} \dot{q}_{\text {net }}+\mathcal{D}_{\theta},
\end{aligned}
$$

where $\mathcal{D}_{\mathbf{v}}$ and $\mathcal{D}_{\theta}$ represent diffusion. As discussed above, the diffusion is in the form of Laplacian dissipation (i.e., $v \nabla^{2} \chi$, where $\chi=\{u, v, \theta\}$ and $v$ is the constant dissipation coefficient) in the baroclinic wave test case, while it is in the form of a $(\mathfrak{n}=2)$ Shapiro filter in the diabatic test case. Note that, in the latter test case, $\mathfrak{n}=2$ gives the best performance in terms of angular momentum conservation in the default CS grid setting. Note also that, when solving the primitive equations in CS grid, a vector-invariant form of equation (11a) must be used to avoid explicit representation of geometry-dependent metric terms. As in the pseudospectral model cores, the equation set is closed by the ideal gas law and the following boundary conditions:

$$
\omega=0 \quad \text { at } \quad p=0, p_{\mathrm{r}} .
$$

Thus, the upper and lower boundaries act as a solid boundary.

\footnotetext{
${ }^{15}$ At this resolution, timestep size of typically 5 times smaller than that used in pseudospectral cores is required to prevent blow-up in the MITgcm core in the default configuration.
} 


\subsection{Test Cases}

The dynamical cores described in Section 2.1 are subjected to three tests, which increase in physical complexity. The test cases are as follows:

1) Steady-State - assesses the ability of the core to maintain a steady-state. A steady-state is often observed in hot extrasolar planet simulations in some parameter regimes. The state in this case is a 'neutrally-stable'16, zonally-symmetric jet, which is nonlinearly balanced with the background temperature. This is an exact solution to the steady-state primitive equations. In theory, when initialized thus, the cores should maintain the state without any change for all times, in the absence of external perturbation. In practice, gravity waves and model truncation errors degrade the steady-state solution over time (Polichtchouk and Cho, 2012). A noticeable deviation from the initial condition implies the presence of numerical and/or programming errors.

2) Baroclinic Wave - assesses the ability of the core to faithfully capture the nonlinear evolution of a well-studied, three-dimensional flow structure (e.g., Simmons and Hoskins, 1979; Thorncroft et al., 1993; Polvani et al., 2004; Jablonowski and Williamson, 2006; Polichtchouk and Cho, 2012). In contrast to the steady-state case, a small perturbation is introduced to the neutrally-stable jet to trigger a baroclinic instability, and subsequent evolution over a finite duration (20 planetary rotations) is followed. The magnitude (but not the sense and precise location) of the jet is typical of that observed in hot extrasolar planet simulations. The primary aim of this test is to clearly expose phase and amplitude errors, which can often be obscured by complicated flow evolutions that cannot be readily compared with analytic solutions. Note that, for this setup, analytic solutions do not exist.

3) Diabatic Forcing - assesses the performance of the core in a setup similar to that used in many hot extrasolar giant planet studies in the past (e.g., Showman et al., 2009; Rauscher and Menou, 2010; Thrastarson and Cho, 2010). In the setup, the effect of zonally asymmetric heating from the host star is idealized as a simple 'Newtonian relaxation' to a prescribed temperature distribution in equation (1b). Subject to this applied diabatic forcing, the atmosphere is 'spun-up' from an initial state of rest. The 'strength' of the forcing is controlled by the specified daynight temperature gradient and characteristic relaxation time which varies in height. The purpose of the test is to elucidate large-scale flow and temperature distributions observed in current simulations of tidally synchronized extrasolar planets. In general, the established flow and temperature distributions can be variable, depending on

\footnotetext{
${ }^{16}$ In the sense that the jet is stable only in the absence of a perturbation destabilizing quickly otherwise.
}

the forcing and dissipation parameters used (e.g., Cho et al., 2008; Thrastarson and Cho, 2011).

Before presenting the test case results, a brief discussion concerning convergence is in order. Throughout the paper we extensively use the word, 'convergence', but take particular care to distinguish three different types of convergence: numerical, visual, and qualitative. In our heuristic definition, numerical convergence is achieved when a model output is the same up to a specified decimal precision (e.g., two places), at least at two different spatial resolutions. This is the most stringent criterion for convergence and not easily achieved if the model parameters (e.g., dissipation coefficient) are different between two resolutions, even for the same dynamical core. Visual convergence is less stringent than numerical convergence and is achieved when plots of the model solutions at two or more resolutions are nearly visually indistinguishable. Qualitative convergence is the least stringent definition and is achieved when the model results at two or more resolutions are similar in a qualitative sense. Solutions which differ in phase and amplitude at a given time, are qualitatively converged if they behave similarly over a finite time window. Qualitative convergence can be achieved within a single core (e.g., at different resolutions) and across different cores (despite different model parameters).

\section{Results}

\subsection{Test Case 1 (TC1): Steady-State}

\subsubsection{TC1 Setup}

In this test case, a nonlinearly balanced, midlatitude eastward jet is specified as the initial condition. The jet is a neutrallystable solution to equations (1), and is unstable in the presence of a perturbation. The setup is identical to the midlatitude jet setup in Polichtchouk and Cho (2012). We review the setup in $p$-coordinate only here. The equivalent setup in $\sigma$-coordinate can be obtained by using the relation, $p=\sigma p_{s}$. The initial zonal flow $u_{0}$ is as follows:

$$
u_{0}(\phi, p)= \begin{cases}U \sin ^{3}\left[\pi\left(\sin ^{2} \phi\right)\right] F\left(z^{*}\right), & \phi \geq 0 \\ 0 \quad, & \phi<0 .\end{cases}
$$

Here, $z^{*}=-H \log \left(p / p_{\mathrm{r}}\right)$ and

$$
F\left(z^{*}\right)=\frac{1}{2}\left[1-\tanh ^{3}\left(\frac{z^{*}-z_{0}}{\Delta z_{0}}\right)\right] \sin \left(\frac{\pi z^{*}}{z_{1}}\right) .
$$

The values of the parameters are: $U=500 \mathrm{~m} \mathrm{~s}^{-1}, z_{0}=$ $1823 \mathrm{~km}, z_{1}=2486 \mathrm{~km}, \Delta z_{0}=414 \mathrm{~km}, H=580 \mathrm{~km}$, and $p_{\mathrm{r}}=10^{5} \mathrm{~Pa}\left(=10^{3} \mathrm{hPa} \approx 1\right.$ bar $)$.

The basic state temperature, $T_{0}=T_{0}(\phi, p)$, is obtained by combining meridional momentum and hydrostatic balance equations - the meridional component of equation (1a) and equation (1c), respectively:

$$
\frac{\partial T_{0}}{\partial \phi}=-\frac{H}{\mathcal{R}}\left(R_{p} f+2 u_{0} \tan \phi\right) \frac{\partial u_{0}}{\partial z^{*}} .
$$


Integrating equation (15) numerically results in a temperature distribution that is in gradient-wind balance with the specified jet (equation (13)). Here, we have used a reference temperature of $1500 \mathrm{~K}$ as the constant of integration. The value is consistent with initial conditions and results of many extrasolar planet GCM calculations. In Fig. 1, the meridional cross-section of the zonally-symmetric basic state flow $u_{0}$ and potential temperature $\theta_{0}$ (left), as well as the longitude-latitude map of the relative vorticity $\zeta_{0}$ at $975 \mathrm{hPa}$ level (right), are shown.

Note that, in the above setup, the jet strength is considerably weaker than in the analogous setup of Polichtchouk and Cho (2012). In this work, weaker jet amplitude is chosen in order to achieve better numerical and visual convergence at lower resolutions. Higher resolution is often required for convergence of high amplitude jets, due to the stronger ageostrophy associated with high speed jets (e.g., Polichtchouk and Cho, 2012).

We emphasize that the initial condition is trivial to set up in all the models-except for the MITgcm in CS grid configuration. To specify the initial zonal wind field in this grid, a MATLAB routine (supplied with MITgcm) is used to re-grid the wind from the LL grid to the CS grid. This re-gridding procedure involves changing the orientation of the wind velocity vector from the LL grid to the CS grid by rotating the vector components through grid orientation angles. As a result of the procedure, small values of meridional wind are artificially introduced in the initial wind field; hence, the initial state becomes slightly less well balanced than that before the re-gridding. However, the re-gridding procedure itself does not cause the destabilization of the jet. We have checked this by regridding the field from the CS grid onto the Gaussian grid: the jet in the Gaussian grid is stable when the unbalancing effects from the corner points in the CS grid are removed.

The steady-state case (as well as the other test cases) have been performed mainly at three different horizontal resolutions. The vertical domain is resolved by 20 equally spaced $p$ or $\sigma$ levels such that the bottom level midpoint is placed at $p=$ $975 \mathrm{hPa}(\sigma=0.975)$ and top level midpoint is placed at $p=$ $25 \mathrm{hPa}(\sigma=0.025)^{17}$. The pseudospectral resolution in the horizontal direction is up to T170 in BOB and up to T85 in other model cores. Here, the letter ' $\mathrm{T}$ ' refers to the triangular truncation and the number refers to the maximum total (as well as the zonal) wavenumber retained in the spherical harmonic expansion. The highest horizontal resolution in MITgcm for the LL grid is G128 and for the CS grid is C64. ${ }^{18}$ The 'G128' designation refers to $256 \times 128$ grid points covering the surface of the sphere. The 'C64' designation refers to $64 \times 64$ points covering one of the six cube faces, for a total of $6 \times 64 \times 64$ points covering the entire surface of the sphere. All the other model specific parameters needed for reproducing the steady-state test case are listed in the Appendix, in Tables A.1-A.3.

\footnotetext{
${ }^{17}$ Note, however, that the bottom interface is placed at $p=10^{3} \mathrm{hPa}(\sigma=1)$ and the top interface at $p=0 \mathrm{hPa}(\sigma=0)$, respectively.

${ }^{18}$ The C64 and C16 CS grids have been generated by us with MATLAB routines provided by MITgcm support. However, the default C32 grid (also MATLAB generated) comes included with MITgcm — hence, we have not generated the $\mathrm{C} 32$ grid ourselves.
}

The dynamical cores are integrated for $20 \tau$, where $\tau$ is one planetary rotation (i.e., $2 \pi / \Omega$ ), with no forcing and dissipation. Hence, $\mathcal{F}_{\mathbf{v}}=\mathcal{D}_{\mathbf{v}}=\mathcal{D}_{\theta}=\dot{q}_{\text {net }}=0$ in equations (1). Note that, in the absence of forcing and dissipation, all dynamical cores should conserve mass, total energy (TE), total angular momentum (AM) and potential temperature exactly. The TE and AM are defined as:

$$
\begin{aligned}
\mathrm{TE} & =\int_{V}\left(\frac{u^{2}+v^{2}}{2}+c_{p} T+\Phi\right) \mathrm{d} M \\
\mathrm{AM} & =\int_{V}\left[\left(\Omega R_{\mathrm{p}} \cos \phi+u\right) R_{\mathrm{p}} \cos \phi\right] \mathrm{d} M,
\end{aligned}
$$

where the integral is taken over the volume $V$ of the atmosphere. Note also, AM is the total absolute angular momentum.

\subsubsection{TC1 Results}

As discussed earlier, all models are expected to maintain the initial condition (Fig. 1) because it is an exact solution to equations (1) in the steady state and there are no external perturbations. However, in practice the initial state degrades over time because balance is never perfectly achieved due to the slight numerical errors generated in the integration of equation (15), as well as in the inherent space and time discretizations. We quantify the numerical errors via two $l_{2}$ error norms: the 'symmetry' norm and the 'degradation' norm (see, e.g., Jablonowski and Williamson, 2006). In TC1, these norms are computed for the zonal wind field $u$.

The symmetry norm assesses the deviation from zonal symmetry (related to eddy kinetic energy) at each instant. It is defined:

$$
\begin{aligned}
& l_{2}[u(t)-\bar{u}(t)]= \\
& \left\{\frac{1}{4 \pi} \int_{s_{\mathrm{B}}}^{s_{\mathrm{T}}} \int_{-\frac{\pi}{2}}^{\frac{\pi}{2}} \int_{0}^{2 \pi}[u(\lambda, \phi, s, t)-\bar{u}(\phi, s, t)]^{2} \cos \phi \mathrm{d} \lambda \mathrm{d} \phi \mathrm{d} s\right\}^{1 / 2} \\
& \approx\left\{\frac{\sum_{k} \sum_{j} \sum_{i}\left[u\left(\lambda_{i}, \phi_{j}, s_{k}, t\right)-\bar{u}\left(\phi_{j}, s_{k}, t\right)\right]^{2} w_{j} \Delta s_{k}}{\sum_{k} \sum_{j} \sum_{i} w_{j} \Delta s_{k}}\right\}^{1 / 2}
\end{aligned}
$$

Here, overbar $\overline{(\cdot)}$ denotes the zonal average; indices $i, j$, and $k$ are for longitude, latitude, and height, respectively; $s$ denotes generalized height (and is either $p$ or $\sigma$ in all the cores); $w_{j}$ are the Gaussian weights (e.g., Canuto et al., 1988) for the pseudospectral cores or are defined as $w_{j}=\left|\sin \phi_{j+1 / 2}-\sin \phi_{j-1 / 2}\right|$ for MITgcm in LL grid, where the 'half-indices' denote points half way between two neighboring grid points; and, $\Delta s_{k}$ are the layer thicknesses. The degradation norm, on the other hand, assesses the deviation of zonal average from the zonallysymmetric initial flow. It is defined:

$$
\begin{aligned}
& l_{2}[\bar{u}(t)-\bar{u}(0)]= \\
& \left\{\frac{1}{2} \int_{s_{\mathrm{B}}}^{s_{\mathrm{T}}} \int_{-\frac{\pi}{2}}^{\frac{\pi}{2}}[\bar{u}(\phi, s, t)-\bar{u}(\phi, s, 0)]^{2} \cos \phi \mathrm{d} \phi \mathrm{d} s\right\}^{1 / 2} \\
& \approx\left\{\frac{\sum_{k} \sum_{j}\left[\bar{u}\left(\phi_{j}, s_{k}, t\right)-\bar{u}\left(\phi_{j}, s_{k}, 0\right)\right]^{2} w_{j} \Delta s_{k}}{\sum_{k} \sum_{j} w_{j} \Delta s_{k}}\right\}^{1 / 2} .
\end{aligned}
$$



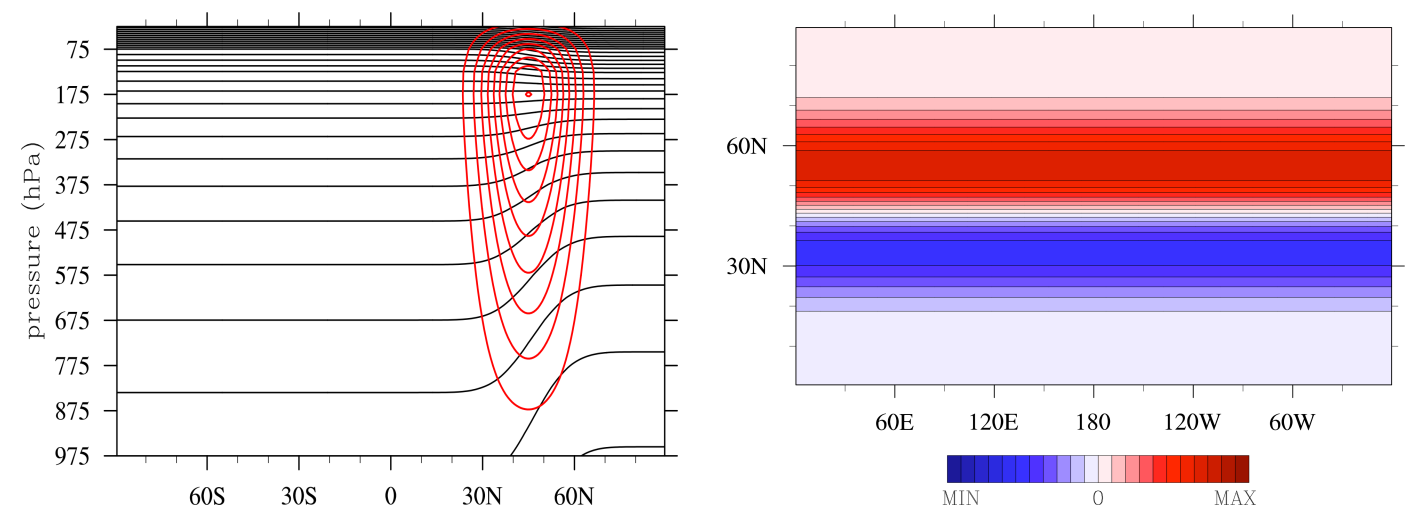

Figure 1: Left: The basic state zonal wind $u_{0}\left[\mathrm{~m} \mathrm{~s}^{-1}\right]$ (red) and potential temperature $\theta_{0}[\mathrm{~K}]$ (black), as a function of latitude and pressure for test case 1, the steady-state test. The contour interval for zonal wind is from $50 \mathrm{~m} \mathrm{~s}^{-1}$ to $500 \mathrm{~m} \mathrm{~s}^{-1}$, in steps of $50 \mathrm{~m} \mathrm{~s}^{-1}$. The contour interval for potential temperature is from $1400 \mathrm{~K}$ to $4400 \mathrm{~K}$, in steps of $100 \mathrm{~K}$. The same setup is used as the initial condition in test case 2, the baroclinic wave test. Right: Basic state relative vorticity ( $\zeta)$ field $\left[\mathrm{s}^{-1}\right]$ as a function of longitude and latitude in cylindrical-equidistant view, centered on the equator, at the $975 \mathrm{hPa}(\approx 975 \mathrm{mbar})$ pressure level. Maximum and minimum values are $+5 \times 10^{-7} \mathrm{~s}^{-1}$ and $-5 \times 10^{-7} \mathrm{~s}^{-1}$, respectively, with contour interval of $5 \times 10^{-8} \mathrm{~s}^{-1}$.

Note, simulation results are interpolated onto a regular LL grid to compute both $l_{2}$ norms for MITgcm in CS grid.

In this work, we have found that all pseudospectral cores and MITgcm in LL grid maintain zonal symmetry to machine precision, at all resolutions. The results are not shown, since they are identical to Fig. 1. However, this is not the case for MITgcm in CS grid: eight special 'corner points' (four in the northern hemisphere and four in the southern hemisphere), where the cube facets meet, introduce an artificial wavenumber- 4 disturbance, quickly degrading the zonal and temporal symmetry. This is shown in Fig. 2.

In the figure, the left panel shows the $l_{2}$ symmetry norm. The effect of the corners is more pronounced at higher horizontal resolution, as the grid size near the corners becomes smaller. The numerical noise introduced by the corner points causes the higher resolution simulations to crash earlier-at $t=15.5 \tau$ and $t=4.5 \tau$ at C32 and C64 resolutions, respectively. Recall that explicit diffusion is not used in these simulations; but with 'enough' diffusion applied, crashing can be prevented (see, e.g., Section 3.2). This is a simple example of when viscosity, filters, or 'fixers' can unintentionally obscure issues in the numerics and when systematic model intercomparisons can be very fruitful. The right panel shows the relative vorticity $(\zeta)$ field from the MITgcm CS simulation at C64 resolution. The field at the $975 \mathrm{hPa}$ level is shown in the cylindrical-equidistant projection, centered on the equator; the time of the simulation is $t=2.5 \tau$. Only the northern hemisphere is shown. The numerical noise from the four special corner points in this hemisphere can clearly be seen. ${ }^{19}$ We have verified that the noise is not due to the slight imbalance of the flow field introduced by the vec-

\footnotetext{
${ }^{19}$ Here, one could argue that this test case (and the next one) unfairly favors the Gaussian and LL grids because the jet is zonal and passes over the corner points. However, rotating the grid does not fully resolve the adverse effect of the corner points nor improves (or reduces the disparity in) the performance of the model over a finite duration (see, e.g., Lauritzen et al., 2010). Note that the default setting of the MITgcm in CS grid is the unrotated grid configuration.
}

tor component rotation, mentioned above (Section 3.1.1). All other models, including MITgcm in LL grid, maintain the initial $\zeta$ distribution throughout the duration of simulation $(20 \tau)$.

Fig. 3 shows the $l_{2}$ degradation norm evolution over the $20 \tau$ duration for all models at varying horizontal resolutions. The degradation norm presents a more stringent quantification of the error growth and fluctuation, as well as the intra-core convergence with resolution, since the deviation is measured against the initial state. We first discuss the error growth and fluctuation characteristics. This is then followed by a discussion of the convergence characteristics.

In the figure, the error growths for the pseudospectral cores and the MITgcm core in LL grid cease, after an initial increase. The initial error growth is due to generation of gravity waves, as already discussed. The error growth characteristics are identical in BOB and PEQMOD cores (top row in Fig. 3), which employ the same vertical discretization scheme. The IGCM and CAM cores (middle row in Fig. 3) exhibit similar error growth characteristics as BOB and PEQMOD, but follow much more closely between themselves. This is not surprising since IGCM and CAM both use the $\sigma$-coordinate vertical discretization. ${ }^{20}$ Note that both IGCM and CAM show errors saturating at slightly higher levels and with larger deviations from the saturation level, compared to PEBOB and PEQMOD.

The MITgcm in the two grids tested, LL and CS grids, show interesting behavior. In the LL grid, the core exhibits similar behavior as the pseudospectral cores-particularly at the higher grid resolutions (see bottom left panel in Fig. 3). At the low grid resolution (G32), the error saturation level and/or fluctuation magnitude are larger than in the pseudospectral cores. How-

More importantly, the grid has not been rotated in past simulations of extrasolar planets using the MITgcm in CS grid. For these reasons, we focus on the unrotated grid configuration in this study.

${ }^{20}$ We remind the reader that the CAM core normally uses the more general $\eta$-coordinate (see, e.g., Thrastarson and Cho, 2010), but it has been run in the simpler $\sigma$-coordinate to facilitate equatable comparison. 

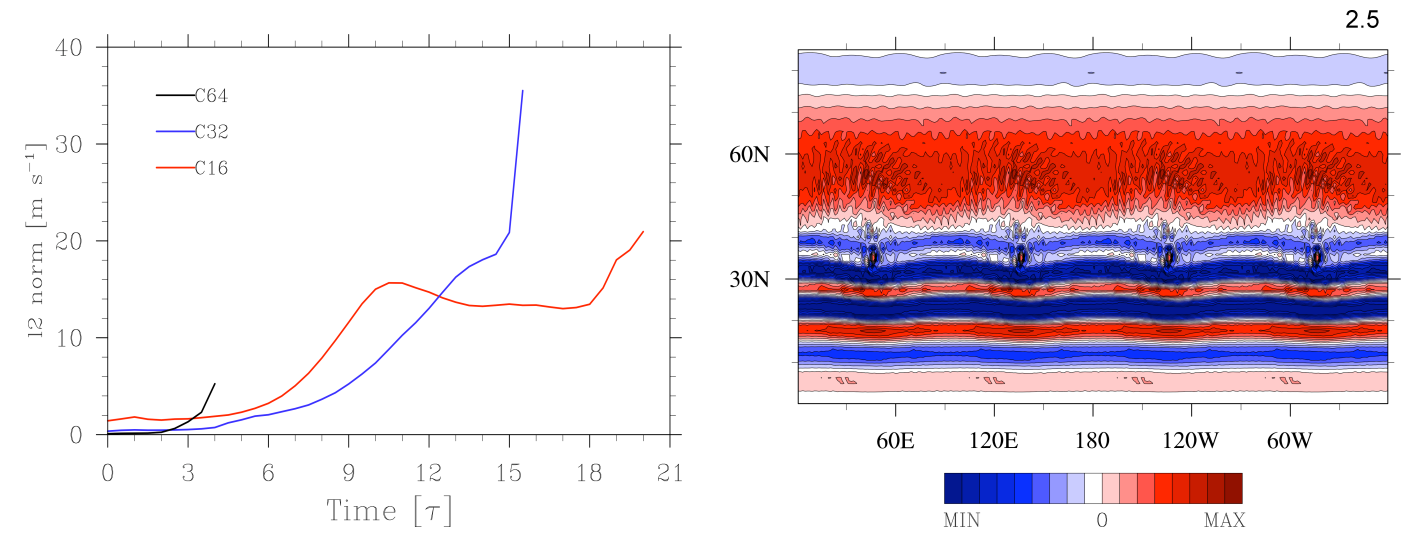

Figure 2: Left: Symmetry deviation $l_{2}$-norm of $[u(t)-\bar{u}(t)]\left[\mathrm{m} \mathrm{s}^{-1}\right]$ for MITgcm cubed-sphere (CS) steady-state case simulations in the default configuration. Three different resolutions (C16, C32, C64) are presented. Higher resolution simulation norms blow-up earlier. Right: $\zeta$ field at $t=2.5 \tau$, at the $975 \mathrm{hPa}$ level, from the C64 resolution simulation in the left panel. Maximum and minimum values are $+6 \times 10^{-7} \mathrm{~s}^{-1}$ and $-6 \times 10^{-7} \mathrm{~s}^{-1}$, respectively, with contour interval of $8 \times 10^{-8} \mathrm{~s}^{-1}$. The increase in the $l_{2}$-norms in the left panel are caused by the special corner points, seen in the right panel. Note, the norms are not exactly zero initially, especially at low resolution. This is due to the errors introduced by the re-gridding procedure of $u$ from LL to CS grid.

ever, both the level and fluctuation magnitude decrease with higher resolution. In contrast, the core in CS grid exhibits error growth behavior that is completely different than any of the cores tested (see bottom right panel in Fig. 3). This is expected from the result already presented in Fig. 2. The degradation error in the MITgcm in CS grid continues to grow with timeagain, due to the wavenumber- 4 noise from the corner points in the CS grid. This effect is probably not so important in simulations of hot extrasolar planet atmospheres, which are strongly forced non-zonally as well as strongly dissipated. However, it could have a deleterious influence on steady state and instability calculations, as demonstrated here (and in the next test case).

Fig. 3 also shows the convergence characteristics of the cores. As can be seen, the pseudospectral calculations are all visually converged. CAM calculations are particularly well converged: the norms for three resolutions tested show essentially no discernible differences. On the other hand, the calculations with the MITgcm in LL grid are not visually converged for resolutions lower than G128, and this is reflected in the figure (bottom left panel). Therefore, these particular calculations are not intra-model converged.

The above behavior is consistent with the theoretical understanding of pseudospectral and finite difference and volume methods and past inter-method comparisons (see, e.g., Durran, 1999; Boyd, 2000, and references therein). The larger saturation and fluctuation of the lower grid resolution calculations are likely due to the second-order accurate finite volume method employed. For a smooth flow devoid of shocks or fronts, for example, the resolution of a pseudospectral simulation is equivalent to an order of magnitude higher horizontal resolution than in a finite volume/difference simulation with the same number of degrees of freedom ${ }^{21}$ (e.g., Canuto et al., 1988; Durran, 1999; Boyd, 2000; Thrastarson and Cho, 2011); this is because the or-

\footnotetext{
${ }^{21}$ This also means that, in practice, a finite volume/difference grid should not be compared with a Gaussian grid of a pseudospectral method with the same
}

der of the pseudospectral method approaches infinity exponentially fast with increasing resolution. Note that the MITgcm in CS grid calculations are neither inter- nor intra-converged, as the $\mathrm{C} 16$ and $\mathrm{C} 32$ calculations strongly diverge after $t \approx 7 \tau$ and the C64 calculation crashes before this point at $t \approx 4 \tau$.

Of all the cores tested, BOB and PEQMOD cores maintain the steady state solution the best: their norms level off with the smallest mean value as well as with the smallest root mean square fluctuation from the mean. This is partly due to the exclusion of the external gravity wave mode, a constraint imposed by the 'zero vertically-integrated divergence over the atmosphere' algorithm employed by the two cores (see Section 2.1.1).

In terms of convergence, pseudospectral cores are all converged at T42 resolution for this test case. Differences in the norms, as the horizontal resolution is increased, is hardly noticeable in these cores: their solutions are visually converged. As already mentioned, the CAM solutions show remarkably little difference at different resolutions: they are numerically converged. Numerical convergence is achieved in BOB at T85 resolution; the norms for T85 and T170 resolutions match exactly up to the second decimal place. Again, this is expected, given the exponential convergence property of the pseudospectral method. In contrast, the MITgcm calculations are not visually converged in both LL and CS grid configurations.

In summary, apart from the MITgcm in CS grid, the steadystate condition is well maintained throughout the duration of the calculations by all of the cores. Hence, these calculations are qualitatively inter-model converged for this test case at

number of grid points, as the latter grid is still equivalent to effectively at least three times the resolution of the former grid. The latter point is demonstrated in Fig. 3 (cf. T21 and G32 calculations, for example). Note also that when shocks/fronts are present, all methods have difficulty representing the flow accurately, unless specialized treatments (available in both pseudospectral and finite difference methods) are implemented to specifically deal with the sharp flow structures. 

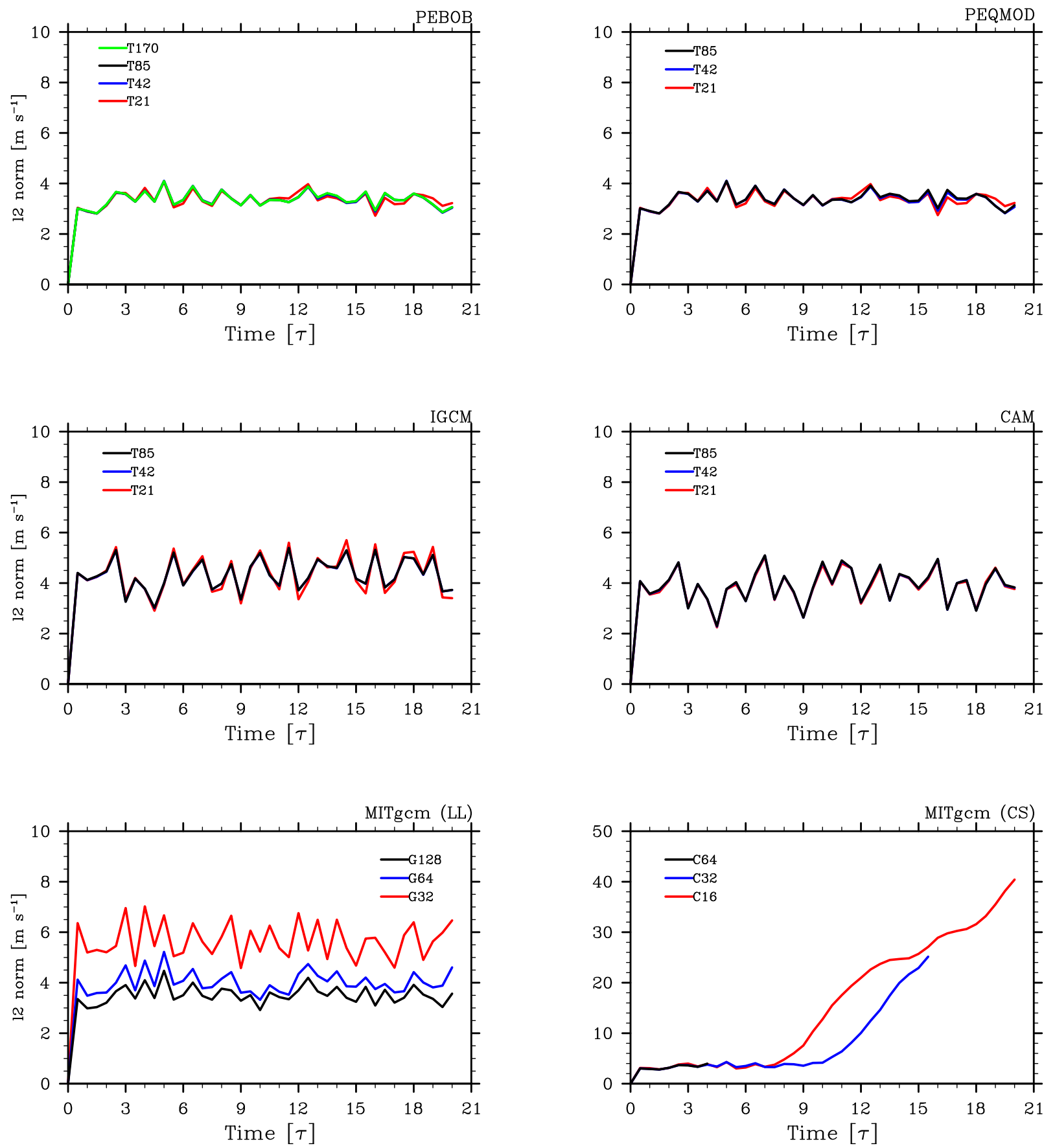

Figure 3: The degradation $l_{2}$-norm of $[\bar{u}(t)-\bar{u}(0)]\left[\mathrm{m} \mathrm{s}^{-1}\right]$ for all the dynamical cores with varying horizontal resolutions. Note that the vertical scale of the plot for the MITgcm in CS grid (bottom right) is five times that of the other panels. In this panel, the three simulations are indistinguishable from each other until just before the C64 simulation crashes, at $t \approx 4.5 \tau$. 
the resolutions considered. As additional measures of convergence, we have verified that these cores conserve the total initial energy (equation (16)) and total angular momentum (equation (17)). The values of the two quantities are $2.3 \times 10^{28} \mathrm{~J}$ and $1.8 \times 10^{32} \mathrm{~J}$ s, respectively, for this test case. These values are maintained throughout the integration to within 0.02 percent (except, of course, for the MITgcm in CS grid). At this point, one may be tempted to down-play the differences between the model cores reported here-particularly in the pseudospectral cores. However, we caution that even such small discrepancies can-and in practice do-lead to non-trivial differences in the model outputs, if the problem is more complex or requires high spatio-temporal accuracy (e.g., instability and transition to turbulence).

\subsection{Test Case 2 (TC2): Baroclinic Wave}

\subsubsection{TC2 Setup}

In this case, an instability is initiated in the neutrally-stable state of Section 3.1 to generate an nonlinearly evolving baroclinic wave. The instability is triggered by perturbing the initial temperature $T_{0}$ with a heat bump $T^{\prime}$ at all pressure levels, where

$$
T^{\prime}(\lambda, \phi)=\operatorname{sech}^{2}(3 \lambda) \operatorname{sech}^{2}\left[6\left(\phi-\frac{\pi}{4}\right)\right] .
$$

Once the instability ensues, the flow is allowed to evolve freely thereafter for $20 \tau$. It is important that exactly this perturbation is used, when attempting to reproduce the results here. This is because, while the flow is expected to asymptotically reach qualitatively the same state, the early-time evolution is different for a different perturbation. It is then difficult to delineate the source of the variations in the subsequent evolution-whether the variations are due to physically different modes being excited or to numerical inaccuracies.

The instability leads to a rapid development of sharp fronts in few planetary rotations (i.e., few $\tau$ 's), and we can no longer integrate the inviscid equations (as in TC1) since there is a rapid build up of energy in small scales. This case is arguably more 'realistic' than the steady-state case, in the sense that explicit viscosity must be used-as in most long-duration simulations involving complex flows.

To make the comparison easier, we choose to implement in this test case a Laplacian dissipation operator $\left(\nabla^{2}\right)$ in all the cores, even though more scale-selective, higher order, hyperdiffusion operators (e.g., $\mathfrak{p} \geq 2$ in equation (5)) are almost always used in pseudospectral calculations. Although hyperdiffusion operators acting on vorticity and divergence fields are common in pseudospectral cores, they are less common in finite volume cores because they are more difficult to implement in the finite volume discretization scheme. In the latter type of cores, alternative strategies are used to effect high scale-selectivity. As discussed in Section 2.1.2, in addition to the harmonic (secondorder) and biharmonic (fourth-order) diffusion, the MITgcm also supports the Shapiro filter.

As in Polvani et al. (2004) and Polichtchouk and Cho (2012), the same value of dissipation coefficient $\left(v_{2}=2 \times 10^{7} \mathrm{~m}^{2} \mathrm{~s}^{-1}\right)$ is used for all the resolutions in TC2. The usual practice is to adjust - or tune - the value for each resolution, problem and model (see, e.g., discussions in Thrastarson and Cho (2011) and Polichtchouk and Cho (2012)). However, $v_{2}$ is not adjusted in this case so that each mode, up to the truncation wavenumber, experiences the same amount of dissipation, regardless of the resolution. For example, the dissipation time at the T21 truncation scale for HD209458b corresponding to the above value of $v_{2}$ is: $\tau_{\mathrm{d}}=3.58 \tau$. In comparison, current flow modeling studies of hot giant extrasolar planets employ a much shorter damping time of $\tau_{\mathrm{d}} \sim 0.02 \tau$ (e.g., Rauscher and Menou, 2010; Heng et al., 2011); hence, these simulations are more dissipative than the ones in this study. However, the damping time used is still generally shorter than that used in the previous, similar study by Polichtchouk and Cho (2012) — again, to allow a more equatable comparison between the different cores to be performed.

The highest horizontal resolutions investigated in this test case are the same as in the steady-state test case (TC1). The resolution specifications and other parameters needed for reproducing this test case are listed in the Appendix, Tables A.4-A.6. Note, unlike in TC1, the true solution to the primitive equations is unknown for this test case.

\subsubsection{TC2 Results}

Fig. 4 shows the evolution of $\zeta$ at the $p=975 \mathrm{hPa}$ surface from a simulation with BOB at T170L20 (i.e., T170 horizontal resolution with 20 vertical levels) resolution, for $t=6 \tau$ to $t=18 \tau$. The $975 \mathrm{hPa}$ pressure surface is chosen because the maximum eddy activity of the unstable evolution occurs near the lower boundary (e.g., Polichtchouk and Cho, 2012). In the evolution, the perturbed jet undergoes a period of linear growth $(t \ll 9 \tau)$, when the most unstable mode (mode 3-4) emerges. By $t \approx 14 \tau$ the evolution is well in its nonlinear stage, characterized by the exponential growth of eddy kinetic energy and wave breaking. The $\zeta$ perturbation exhibits a distinct northwestsoutheast tilt on the poleward side of the jet and southwestnortheast tilt on the equatorward side of the jet. Near $t=18 \tau$, the eddy kinetic energy reaches the maximum value of the simulation and the barotropic decay cycle, in which eddy kinetic energy is returned back to the mean flow, ensues for $t \gtrsim 20 \tau$. In this period, the cyclones (areas of positive $\zeta$ anomalies, shown in red in the figure) that have emerged from the wave breaking, start interacting and advance poleward (e.g., Cho, 2008; Polichtchouk and Cho, 2012).

The evolution presented in Fig. 4 is the high resolution 'reference solution' for the BOB core. For the other pseudospectral cores and the MITgcm core in LL and CS grids, the reference solution is computed at T85L20, G128L20, and C64L20 resolutions, respectively. In addition to these solutions, the outputs from the other model cores may be compared with the T170L20 reference solution obtained with the BOB core. In principle, since all cores solve the same equations (and boundary conditions), the high resolution reference solution computed with one of the cores should serve as a reference solution for all the models. However, in practice, there is a danger in using a single model core to determine the reference solution in problems involving unstable states, as noted by Jablonowski and 
6

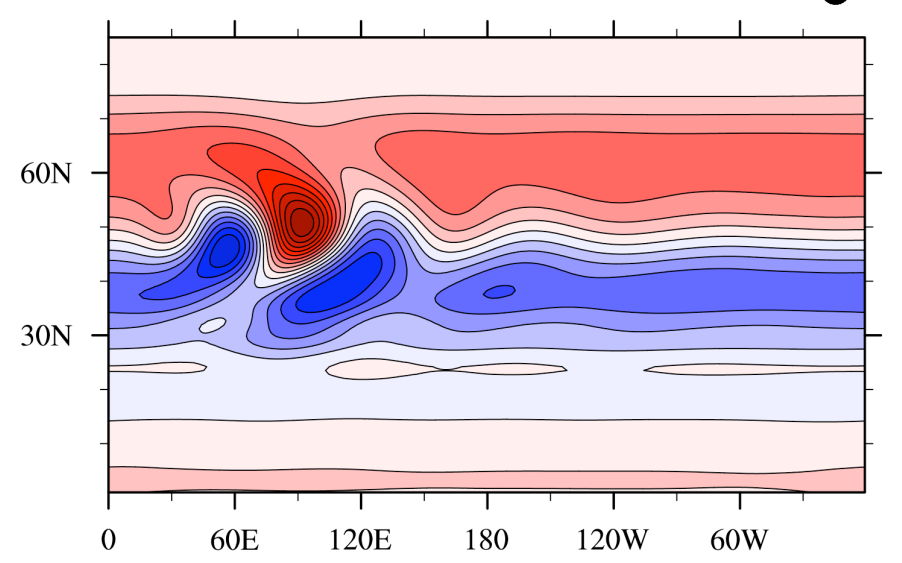

14

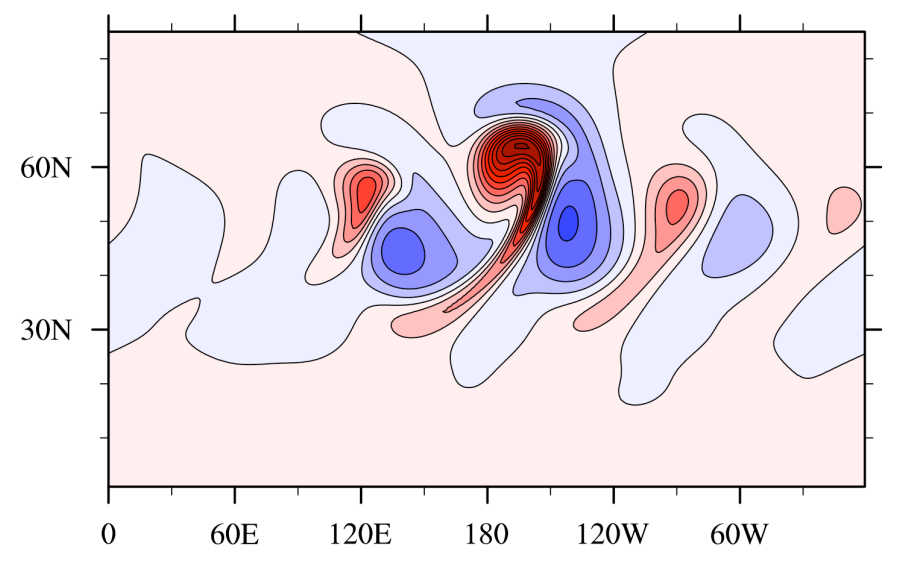

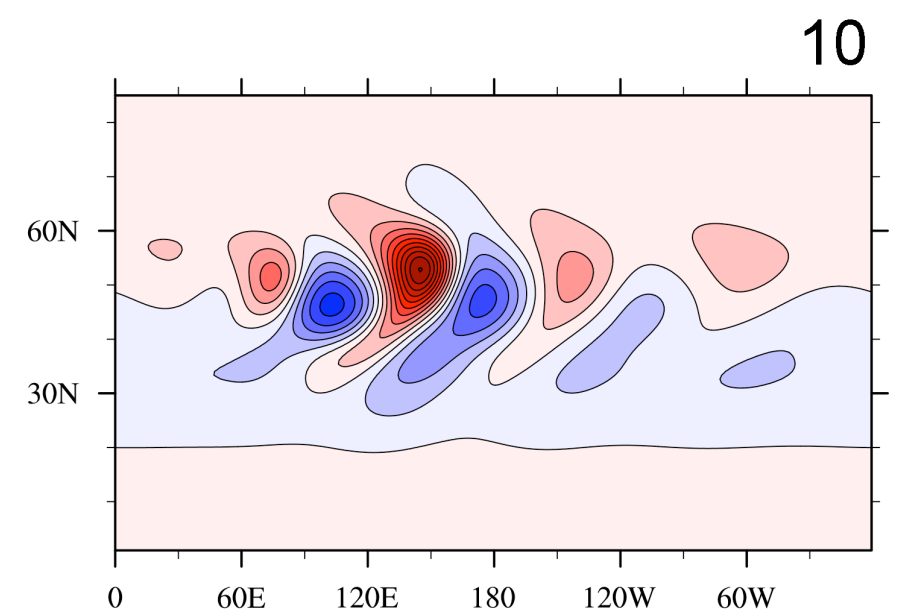

18

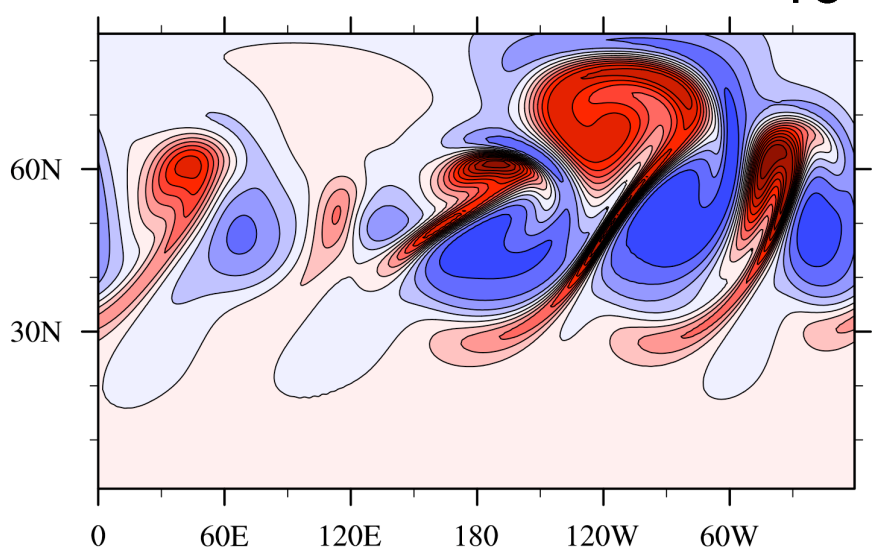

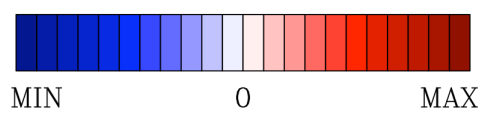

Figure 4: Relative vorticity $(\zeta)$ field from T170L20 run with BOB in cylindrical-equidistant view, centered on the equator. The fields are shown at the 975 hPa pressure level for $t=6 \tau$ to $t=18 \tau$. Maximum and minimum values are: $\pm 1 \times 10^{-6} \mathrm{~s}^{-1}(t=6 \tau) ; \pm 5 \times 10^{-6} \mathrm{~s}^{-1}(t=10 \tau)$; and, $\pm 2 \times 10^{-5} \mathrm{~s}^{-1}(t=14 \tau$ and $t=18 \tau)$. The contour intervals are, respectively, $1 \times 10^{-7} \mathrm{~s}^{-1}, 5 \times 10^{-7} \mathrm{~s}^{-1}$ and $2 \times 10^{-6} \mathrm{~s}^{-1}$. Note the large, an order of magnitude, change in the amplitude of $\zeta$ during the evolution-as well as the formation of sharp fronts and coherent vortices, particularly at $t=14 \tau$ and $t=18 \tau$. 
Williamson (2006). This is due to the differences in how various model cores handle geostrophic adjustment.

Fig. 5 compares the solutions of all the dynamical cores at the T85L20, G128L20, and C64L20 resolutions at $t=10 \tau$ (cf. upper right frame in Fig. 4). As in TC1, the calculations with IGCM and CAM look nearly identical. The same is true for BOB and PEQMOD calculations, although the amplitude of vorticity anomalies in PEQMOD is somewhat stronger than in BOB. Comparing with IGCM and CAM, the amplitude in PEQMOD is noticeably stronger. In general, we have found that the vorticity anomalies are stronger in the $p$-coordinate pseudospectral cores than in $\sigma$-coordinate pseudospectral cores. In contrast, the phases are impressively similar among all the pseudospectral cores.

There are, however, considerable differences in both amplitude and phase between solutions with MITgem and pseudospectral cores. Compare the overall vorticity fields, and especially the magnitude of the $\zeta$ anomalies in the fields. This is partly caused by $\zeta$ not being a prognostic variable in the MITgcm core: $u$ and $v$ fields are evolved, and the calculation of $\zeta$ from these fields introduces some errors. If the potential temperature $(\theta)$ field is compared instead of the $\zeta$ field, the MITgcm LL grid solution resembles the corresponding PEQMOD solution more closely (not shown). However, even using the $\theta$ field, the MITgcm CS grid solution differs significantly from the pseudospectral core solutions. This difference-between the two MITgcm solutions, in LL and CS grids-is revealing (cf. two bottom panels in Fig. 5). Note that the only difference between the two calculations is the grid (and the use of a highwavenumber zonal filter in the LL grid calculation).

The solution from MITgcm in CS grid clearly exhibits a different unstable mode structure than that of the LL grid solution at $t=10 \tau$ (i.e., $\sim 5$ for the CS grid compared with $\sim 4$ for the LL grid). The difference is due to the corners in the CS grid, which provide an additional source of perturbation. We have verified this by running a MITgcm in CS grid baroclinic wave simulation without perturbing the background temperature $T_{0}$ by $T^{\prime}$ (see equation 20). In this case a clear mode- 4 structure associated with the corner points (different from those produced in pseudospectral core calculations shown in Fig. 5) dominates the evolution throughout.

To assess the convergence (with resolution) characteristics of a model, we compute the $l_{2}$ relative vorticity norm at the lowest model layer (i.e., the $\sim 975 \mathrm{hPa}$ pressure surface). The norm is defined as follows:

$$
\begin{aligned}
& l_{2}\left[\zeta\left(s_{20}\right)\right]= \\
& \left\{\frac{1}{4 \pi} \int_{0}^{2 \pi} \int_{-\frac{\pi}{2}}^{\frac{\pi}{2}}\left[\zeta\left(\lambda, \phi, s_{20}, t\right)\right]^{2} \cos \phi \mathrm{d} \phi \mathrm{d} \lambda\right\}^{1 / 2} \\
& \approx\left\{\frac{\sum_{i} \sum_{j}\left[u\left(\lambda_{i}, \phi_{j}, s_{20}, t\right)\right]^{2} w_{j}}{\sum_{i} \sum_{j} w_{j}}\right\}^{1 / 2},
\end{aligned}
$$

where $s_{20}$ is the lowest model layer in $p$ - or $\sigma$-coordinate and the sums are taken over all $\left(\lambda_{i}, \phi_{j}\right)$ points on the sphere. The integration weights are defined as in equation (18). After calculating the $l_{2}\left[\zeta\left(s_{20}\right)\right]$-norm for each resolution, the highest res- olution $l_{2}$-norm is subtracted from lower resolution $l_{2}$-norms within the same model to assess model core convergence.

The differences between $l_{2}\left[\zeta\left(s_{20}\right)\right]$-norms within the same core are shown in Fig. 6. It is clear that none of the cores are (numerically or visually) converged at the second lowest resolution-T42, G64 and C32: the curves all deviate from zero (cf. in the upper left panel the 'T85-T170' curve, which shows visual convergence). The non-convergence is particularly apparent after the baroclinic instability enters the fully nonlinear growth stage $(t \gtrsim 10 \tau)$. Even at T85 resolution, BOB is only visually (not numerically) converged. This can be verified by comparing the top right panel of Fig. 4 to the top left panel of Fig. 5: the plots in the two panels are very close to each other but not identical.

Note that the $y$-scale in the MITgcm plots in Fig. 6 (as well as in Fig. 7) is an order of magnitude larger than in the corresponding pseudospectral core plots. This suggests that the apparent qualitative inter-convergence of the MITgcm core in LL grid at G128L20 resolution (seen in Fig. 5) is suspect. We have verified that large differences in the $l_{2}$-norm in MITgcm are not caused by the errors introduced in the calculation of $\zeta$. For example, the $l_{2}$-norm of the surface $u$-field behaves in a similar way and visual convergence is clearly not achieved in the MITgcm core at the highest resolution tested in this work.

Fig. 7 shows the differences between $l_{2}\left[\zeta\left(s_{20}\right)\right]$-norms of a given core and the T170 $l_{2}\left[\zeta\left(s_{20}\right)\right]$-norm of BOB. Hence, here we are treating the T170 calculation as a 'reference solution'as if it were the 'correct solution'. Recall that ideally calculations by each core should be compared with T170 (or higher) resolution calculation of the same core. For technical reasons, this is not feasible in all the cores. However, this comparison is still useful and provides some insights. The figure clearly demonstrates that solutions of other model cores are not visually converged to BOB's high-resolution solution. In all model solutions, the norms are small until $t \sim 9 \tau$, but then increase markedly once the evolution enters the fully nonlinear stage, when the wave begins to develop sharp fronts. This is expected, given the behavior of baroclinically unstable evolution reported in Polichtchouk and Cho (2012).

Note that the evolution of the baroclinic wave growth in this set of simulations is considerably retarded by the stronger explicit dissipation, compared to that in Polichtchouk and Cho (2012) (recall that Laplacian dissipation, rather than superdissipation, is employed here). Moreover, the initial jet's maximum speed is weaker here than that in Polichtchouk and Cho (2012), leading to a smaller growth rate for the baroclinic instability. Polichtchouk and Cho (2012) have demonstrated that baroclinic instability in a similar situation is only marginally captured with $\mathrm{T} 85$ resolution for a $1000 \mathrm{~m} \mathrm{~s}^{-1}$ jet. Consistent with that study, all pseudospectral cores are visually converged at resolution T85 in this study, as expected for a $500 \mathrm{~m} \mathrm{~s}^{-1}$ jet. The more stringent resolution criterion for convergence in Polichtchouk and Cho (2012) is due to the stronger ageostrophy present in a faster jet, as already discussed in Section 3.1.1. Similarly, if the initial jet amplitude had been greater than $1000 \mathrm{~m} \mathrm{~s}^{-1}$, the resolution at which convergence would be achieved is expected 

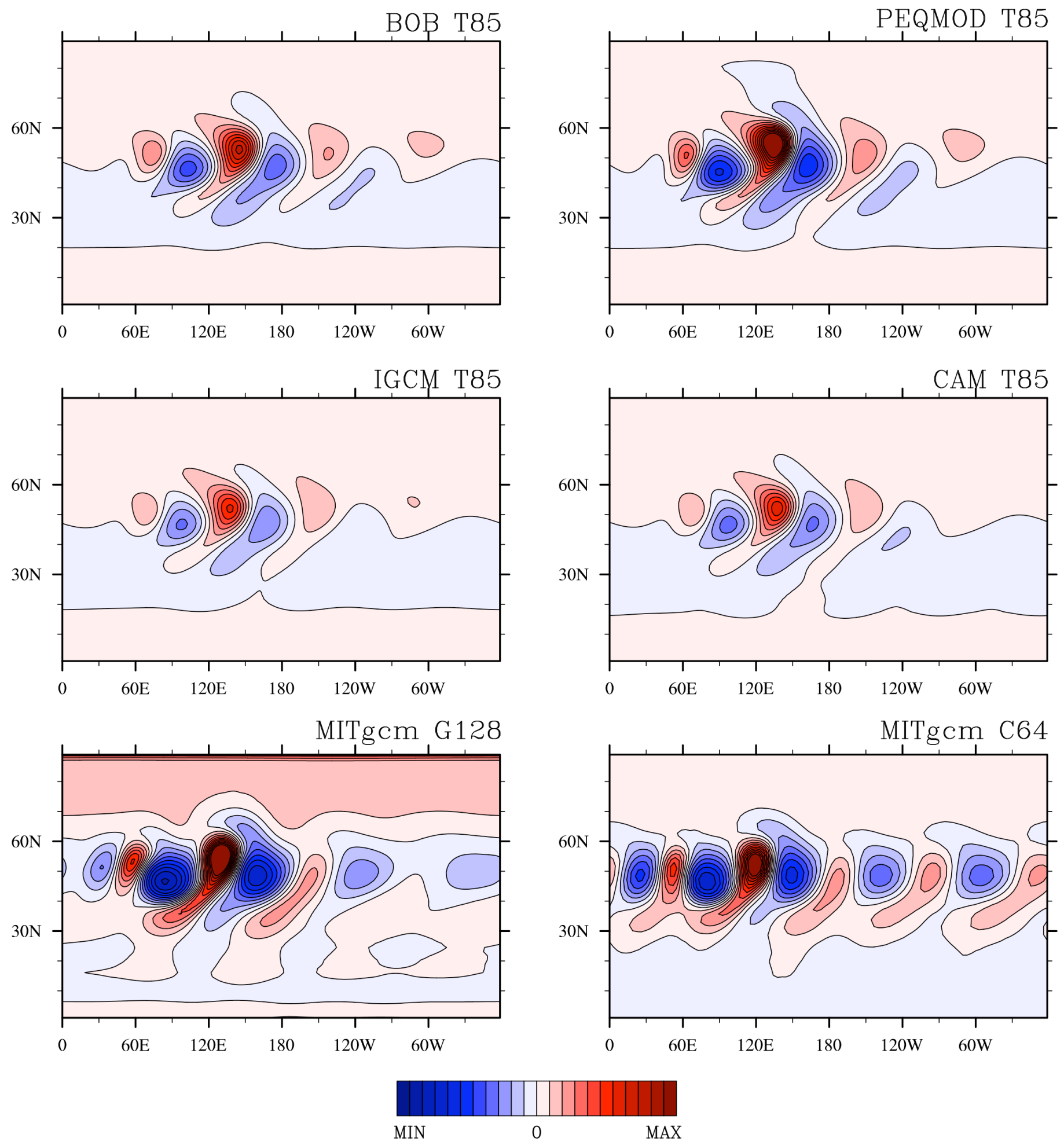

Figure 5: Cylindrical equidistant view, centered on the equator, of $\zeta$ field at $t=10 \tau$ from different model cores. The resolution is the highest tested in all the cores, except in BOB; it is the second highest. The fields shown are from the bottom vertical level ( $\sim 975 \mathrm{hPa})$. Maximum and minimum values for all the cores are $\pm 5 \times 10^{-6} \mathrm{~s}^{-1}$, with contour interval $5 \times 10^{-7} \mathrm{~s}^{-1}$. These fields are to be compared with each other, as well as with that at $t=10 \tau$ in Fig. 4 . 

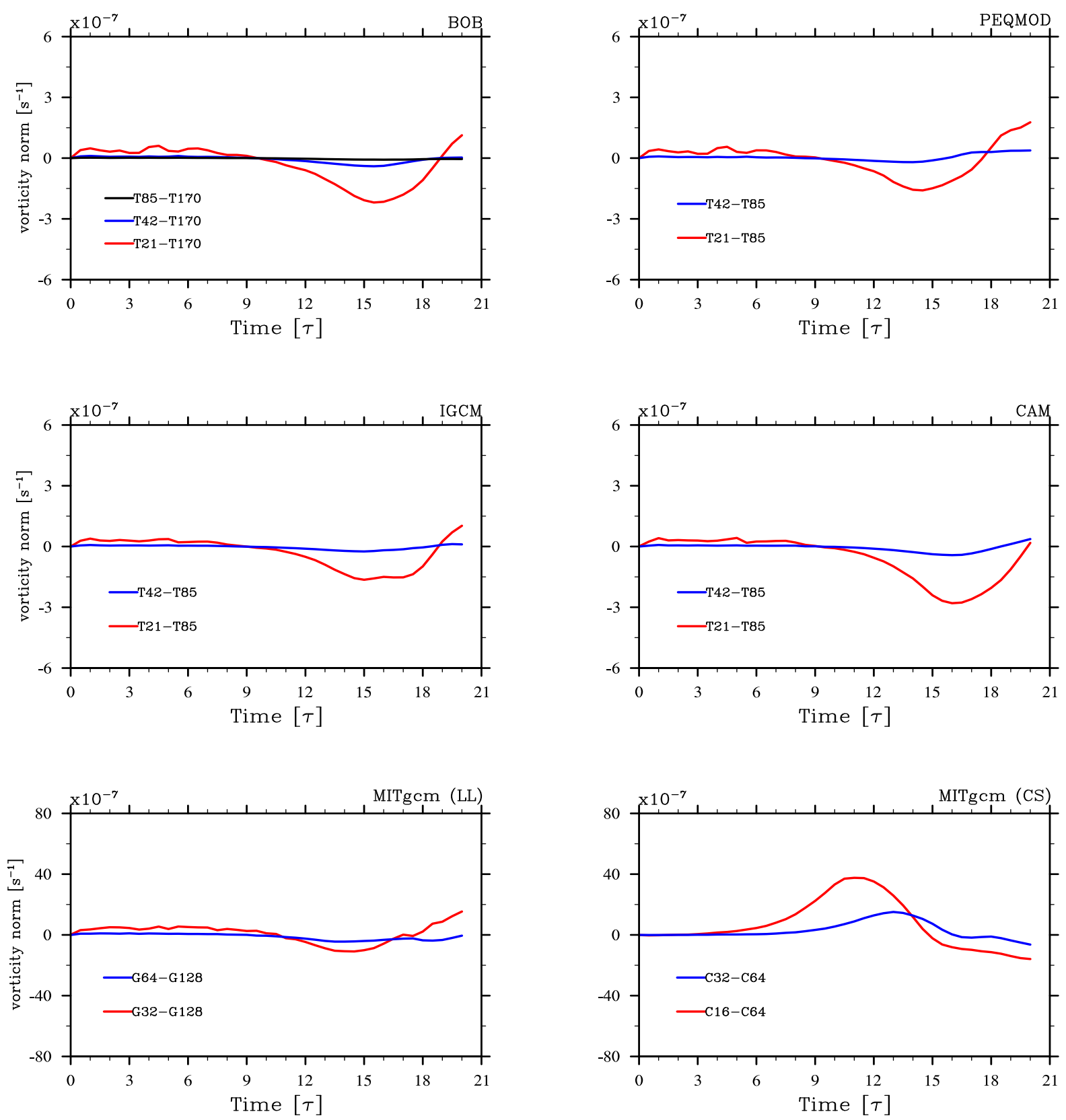

Figure 6: Differences of the root mean square $l_{2}$ vorticity norm $\left[\mathrm{s}^{-1}\right]$ between high resolution reference solution and lower resolution solutions within the same model core. The T85 BOB simulation is well converged. No other cores are 'intra-model' converged. Note, the $y$-scale in the two plots of the bottom row for the MITgcm core is nearly an order of magnitude larger than in the other plots. 

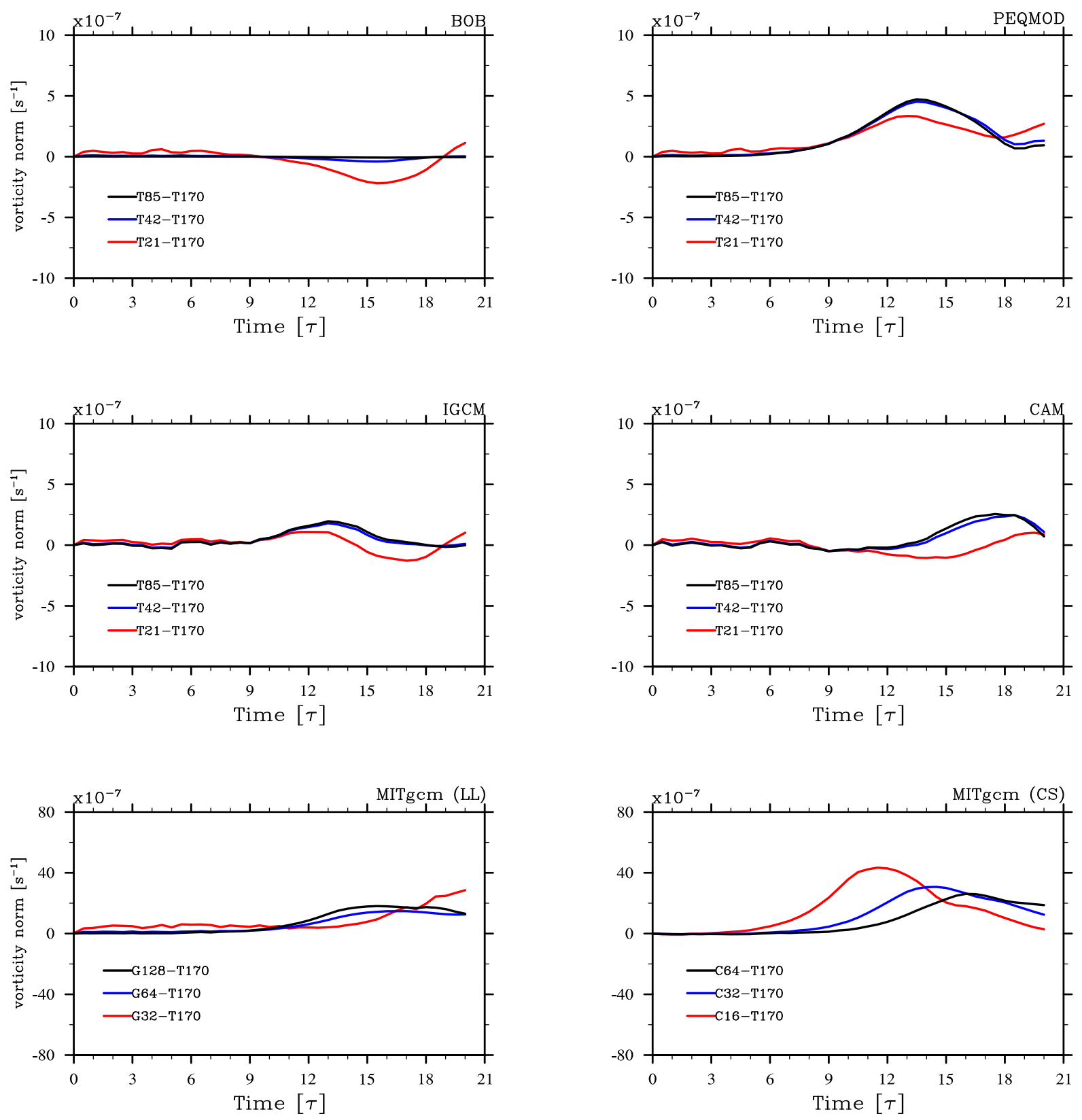

Figure 7: Differences of the root mean square $l_{2}$ vorticity norm $\left[\mathrm{s}^{-1}\right]$ between T170L20 reference solution for BOB and solutions with other dynamical cores at various resolutions. The scales are same as in Fig. 6 . The T42 simulations in all the pseudospectral cores appears to be marginally 'intra-converged'. MITgcm core in both LL and CS grids are not converged, particularly in the latter grid. 
to be correspondingly higher. ${ }^{22}$

Interestingly, even with the application of Laplacian dissipation, wchich is a strong dissipation, the global energy is conserved to within 0.1 percent in all the model cores throughout the integration $(t=20 \tau)$. Global angular momentum is conserved to within 0.02 percent in all the model cores throughout the integration. Note that this is for Laplacian dissipation only, as only this dissipation is used in TC2. These conservation properties should be compared with the corresponding ones in the next test case.

\subsection{Test Case 3 (TC3): Diabatic Forcing}

\subsubsection{TC3 Setup}

No 'physical processes' (e.g., net heating, wave drag, convection) were specified in the setup of TC1 and TC2, if explicit dissipation is not considered to be representing 'turbulent viscosity' in the latter test case. Most of these processes are as yet poorly constrained by observations or unobtainable from first principles for extrasolar planets (see, e.g., discussion in Cho et al., 2008; Cho, 2008; Showman et al., 2010). Two such processes are irradiation from the host star and radiative cooling in the atmosphere of the planet. These processes are currently represented essentially in all extrasolar planet atmosphere simulations in a highly idealized way. For example, Newtonian relaxation parameterization to a prescribed 'equilibrium temperature' is often used to crudely represent the combined thermal forcing (see, e.g., Cooper and Showman, 2005; Showman et al., 2008; Menou and Rauscher, 2009; Rauscher and Menou, 2010; Thrastarson and Cho, 2010, 2011; Heng et al., 2011). Despite the crudeness, here we also use the parameterization - given its simplicity and common usage in past works. The idea is to be reasonably close to past simulations, while facilitating reproducibility of the present work and clean comparisons with future work.

In the Newtonian relaxation parameterization, the source term $\left(\dot{q} / c_{p}\right)$ in the thermodynamic equation (equations $(4 \mathrm{e})$, (7e) or (11d)) is specified as

$$
\frac{\dot{q}}{c_{p}}=-\frac{1}{\tau_{\text {th }}}\left(T-T_{e}\right) .
$$

Here, in its general form, $T_{e}=T_{e}(\lambda, \phi, s, t)$ is the equilibrium temperature and $\tau_{\text {th }}=\tau_{\text {th }}(\lambda, \phi, s, t)$ is the thermal relaxation time. Both $T_{e}$ and $\tau_{\text {th }}$ distributions are currently not well known, both in space and in time. In many studies, very short relaxation times ( $\ll 1$ hour) and large equilibrium temperature gradients across the day-night terminator $(\approx 1000 \mathrm{~K})$ are specified (e.g., Showman et al., 2008; Rauscher and Menou, 2010; Thrastarson and Cho, 2010). Such a condition constitutes an 'extreme forcing' on the dynamics-especially in simulations started from rest state, spun up to a strongly unbalanced state (Cho et al., 2008; Thrastarson and Cho, 2011; Polichtchouk and

\footnotetext{
${ }^{22}$ Note, speeds greater than $2000 \mathrm{~m} \mathrm{~s}^{-1}$ are often produced in many hotJupiter simulations. Some simulations produce speeds which are much greater than this.
}

Cho, 2012): the Solar System planets are characterized by comparatively much longer $\tau_{\text {th }}$ and much smaller $T_{e}$ gradient.

In this test case, $T_{e}$ is height-independent (i.e., $\partial T_{e} / \partial s=0$ ) and both $T_{e}$ and $\tau_{\text {th }}$ are steady (i.e., $\left.\partial\left\{T_{e}, \tau_{\text {th }}\right\} / \partial t=0\right)$ ). In general, both $T_{e}$ and $\tau_{\text {th }}$ are complicated functions of space and time (Cho et al., 2008; Showman et al., 2009). However, in keeping with the overall aim of this work, we choose a setup which is at once easily describable and easily implementable in all models. Here, we choose $T_{e}$ to be as in Thrastarson and Cho (2011):

$$
T_{e}=T_{m}+\Delta T_{e} \cos \phi \cos \lambda,
$$

where $T_{m}=\left(T_{D}+T_{N}\right) / 2$ and $\Delta T_{e}=\left(T_{D}-T_{N}\right) / 2$ with $T_{D}=1900 \mathrm{~K}$ and $T_{N}=900 \mathrm{~K}$ the maximum and minimum temperatures at the day and night sides, respectively. We set $\tau_{\text {th }}$ to vary linearly with pressure (or $\sigma$ ) such that, at the $p=975 \mathrm{hPa}(\sigma=0.975)$ level, $\tau_{\text {th }}=3.6 \times 10^{5} \mathrm{~s}$ and, at the $p=25 \mathrm{hPa}(\sigma=0.025)$ level, $\tau_{\mathrm{th}}=3.6 \times 10^{4} \mathrm{~s}$. The relaxation time is just slightly longer than in some recent studies of hot extrasolar planets, making the forcing slightly less 'violent'. The basic state temperature is isothermal and set to $T=1400 \mathrm{~K}$; and, in all simulations, initial wind $\mathbf{v}_{0}$ is zero everywhere in the computational domain. The vertical domain in all the calculations in this test case, as in the previous test cases, extends from $975 \mathrm{hPa}$ to $25 \mathrm{hPa}$ (again, $\approx 975$ mbar to $\approx 25 \mathrm{mbar}$, respectively). Similarly, horizontal resolutions are the same as in TC1 and TC2 and listed with other model parameters in the Appendix, in Tables A.7-A.9. The calculations are run for $100 \tau$, much longer than the maximum $\tau_{\text {th }}(\approx 1.2 \tau)$.

To control the small-scale noise inherent in all calculations, superdissipation (see equation (5)) is applied in pseudospectral simulations (in each layer) to prevent accumulation of energy at the small scales. Note that this is the 'least common denominator' dissipation, since not all of the tested pseudospectral cores come with higher order (hyper)viscosity as the default. Superviscosity is also more equitably compared to explicit viscosity used in finite volume cores, which in general cannot dissipate as scale-selectively as cores using the pseudospectral algorithm. The value of superdissipation coefficient $v_{4}=10^{22} \mathrm{~m}^{4} \mathrm{~s}^{-1}$ at T85 horizontal resolution (corresponding to a damping time of $190 \mathrm{~s}$ for the smallest resolved scale) is chosen based on the study of Thrastarson and Cho (2011): they have found this value to produce a well-behaved kinetic energy spectrum with the $0.1 \leq\left(\tau_{\text {th }} / \tau\right) \leq 3$ vertical distribution.

In this test case, unlike in TC2 above, the value of $v_{2 \mathfrak{p}}$ is increased/decreased with decreasing/increasing resolution for a given $\mathfrak{p}$ in pseudospectral calculations (see Table A.7); this practice is common in simulation studies. The procedure will definitely preclude numerical convergence as we have defined this convergence in the present work. However, since our simulations are not numerically converged in general in the simpler test cases (particularly in TC2), we do not expect numerical convergence in the more extreme conditions of TC3. Hence, we focus our attention on visual and qualitative convergences in this test case.

In past extrasolar planet simulations performed with MIT$\mathrm{gcm}$, it has been customary to use the Shapiro filter (e.g., Show- 
man et al., 2009; Lewis et al., 2010). Hence, the power-two $(\mathfrak{n}=2$ in equation (10)) Shapiro filter is used here to control oscillations near the grid-scale. Note that the above mentioned studies have used $\mathfrak{n}=4$ Shapiro filter (Showman, private communication). Recall that the strength of the filter is controlled by $\tau_{\text {shap }}$, for a given $\Delta t$ and $\mathfrak{n}$. In this work, the value of $\tau_{\text {shap }}$ is chosen so that $\Delta t / \tau_{\text {shap }}=1 / 6$ for all resolutions. By experimenting with different values, we have found this value to give flow and temperature structures that are qualitatively in good agreement with the the pseudospectral cores across different resolutions: in general, we have found simulations with $\Delta t / \tau_{\text {shap }}=1 / 12$ to be under-dissipated and $\Delta t / \tau_{\text {shap }}=1 / 3$ to be over-dissipated with the model in its default setting.

As discussed in Section 1, past simulations of diabaticallyforced, hot, giant extrasolar planets using different models produce different results - even for fairly similar (but not identical) setup. In many cases, the results are qualitatively different, and the origin of the difference is not obvious. Quite often, this is because all the details of the models, model parameters, and model setup are not reported in the literature-and sometimes not even described in the original model documentation. Hence, truly 'clean', unambiguous comparisons have not been possible thus far. In TC3, the physical setup in all the model calculations is identical. Our aim here is to identify whether variation in recent model results is merely due to the differences in physical setup, or whether variation is also attributed to differences in the numerical formulation of a model (e.g., dissipation scheme, spatial grid, discretization method, etc.).

\subsubsection{TC3 Results}

Fig. 8 shows longitude-latitude maps of the temperature $(T)$ field, with horizontal wind vectors (v) overlaid, from simulations with different model cores. The resolutions for the pseudospectral cores and the MITgcm in LL and CS grids are: T85L20, G128L20, and C64L20, respectively. The instantaneous fields at the $p \approx 475 \mathrm{hPa}$ level at $t=(5 \tau, 20 \tau, 100 \tau)$ are shown. The figure illustrates the main result of this comparison: when subject to strong 'hot-Jupiter-like' forcing, different model cores produce solutions which are visually different among them. This is caused by spatiotemporal variability in the computed fields and renders specific predictions, such as the precise location of hot and cold regions, difficult (see right column of Fig. 8).

Qualitatively, there are some notable common features. For example, most models produce a 'quadrupolar-flow' structure, with two large cyclonic and anti-cyclonic vortex-pairs straddling the equator. The flow in all the simulations is time variable with vortices appearing nearly stationary at times or moving longitudinally eastward or westward at other times, disappearing and reforming on a time scale of 5-7 $\tau$. The temperature in all cases is strongly linked to the flow and varies on corresponding timescales. Consequently, the minimum-tomaximum temperature ranges vary from $\sim 600 \mathrm{~K}$ to $\sim 200 \mathrm{~K}$, at the shown pressure level (e.g., compare middle and right panel in the third row of Fig. 8). Despite the qualitative similarity, model results are quantitatively very different and can diverge more markedly when integrated for longer durations than shown in the figure.

At the beginning, during the first few $\tau$ 's, the flow in all models resembles a linear, 'Matsuno-Gill-type' solution (Matsuno, 1966; Gill, 1980). In the solution, westward-propagating Rossby waves and eastward-propagating Kelvin waves are generated as a response to the specified mode- 1 zonal heating. At high pressure (lower altitude) levels, there is a convergent flow near the substellar point, accompanied by rising motion; concurrently, there is a divergent flow near the antistellar point, accompanied by sinking motion (not shown). At low pressure (higher altitude) levels, there is a divergent flow near the substellar point and a convergent flow near the antistellar point. In the classic Matsuno-Gill setup, strong linear (momentum and thermal) drags balance the forcing. However, in the absence of strong momentum drag, as in this test case, nonlinear interactions quickly degrade the Matsuno-Gill-type solution, and the model solutions start to deviate strongly from the Matsuno-Gill solution-and, importantly, from each other. The latter is due to how different model cores handle adjustment, as discussed earlier.

At early times $(t<10 \tau)$, all core solutions are still similar but a small phase difference is already clearly evident between BOB and PEQMOD (which are nearly identical to each other at this point) and the other model cores (see left column in Fig. 8). At later times $(t \geq 10 \tau)$, all solutions start to visibly diverge from each other and significantly differ quantitatively. For example, the north-south flow symmetry is broken in CAM and PEQMOD simulations (at $t=14 \tau$ ) and in MITgcm in both LL and CS grids (at $t=10 \tau$ ). In contrast, the symmetry is not broken in BOB and IGCM cores even at $t=100 \tau$. It is important to understand that the temporal variability observed here is not due to large-scale baroclinic instability stemming from the location and thermal forcing of the lower boundary. We have visually and quantitatively checked (i.e., eddy production, wave propagation, and heat and momentum fluxes) that large-scale baroclinic instability is not present in this setup and that similar variability is exhibited even with the lower boundary placed much deeper (e.g., 10 and 100 bars) with the forcing limited down to only 1 bar. However, small-scale waves are produced through the adjustment process.

The flow structure remains either quasi-symmetric about the equator throughout the integration (see panels for BOB and IGCM in Fig. 8) or the equatorial flow symmetry is broken at an early time (see panels for PEQMOD, CAM, and MITgcm in Fig. 8). Detailed analyses of the computed fields show that the symmetry breaking is associated with emergence of a large equatorial Rossby wave at $t \approx 10 \tau$, which is not as prominent in simulations with BOB or IGCM. The north-south symmetry in these simulations is not an artifact of a short integration time. It remains even at the end of a $2000 \tau$ simulation with BOB at T21L20 resolution (not shown). However, when the simulation with BOB is initialized with a small, random perturbation in the flow, the north-south symmetry does break at an early time and the flow and temperature evolution closely match simulations with PEQMOD. A likely explanation of the equatorial symmetry breaking is errors introduced by insufficient precision. By repeating this test case with PEQMOD at 

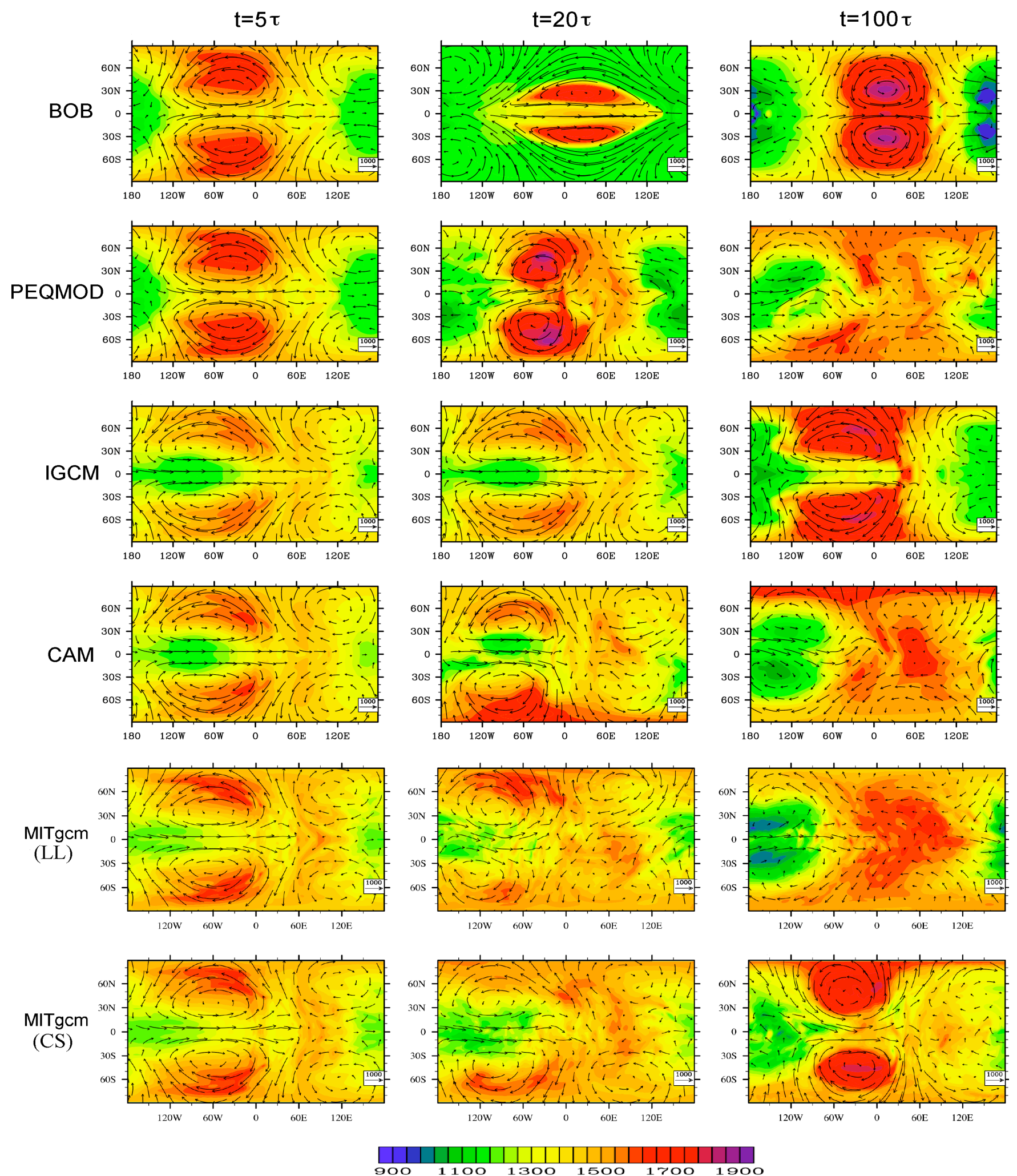

Figure 8: Temperature (color coded in $\mathrm{K}$ ) with wind vectors overlaid, for the diabatic forcing test case (TC3) with different model cores, at three times at the $475 \mathrm{hPa}$ level. Form left to right, the snapshots are taken at $t=(5 \tau, 20 \tau, 100 \tau)$. The (top, second, third, fourth, fifth, bottom) row is, respectively, from a simulation with (BOB, PEQMOD, IGCM, CAM, MITgcm in LL grid, MITgcm in CS grid). The resolution for pseudospectral codes is T85L20 and for MITgcm in LL and CS grids is G128L20 and C64L20, respectively. The flow and temperature distributions are qualitatively similar (e.g., quadrupolar flow) but quantitatively different (e.g., time-variable). 
single, double and quadruple precisions, we have found that the onset time of equatorial symmetry breaking roughly doubles every time the precision is doubled, in this experiment.

Since the flow and temperature structure is strongly time variable in all the simulations, snapshots in time may give an incomplete-possibly even misleading - picture, since large differences in Fig. 8 could simply be due to 'phasing' (simple translation of the flow structure in time). To quantify variability and the behavior with resolution, a time series of global average temperature is shown in Fig. 9. The first thing to note is that essentially all the simulations are equilibrated in temperature: there are no secular growth or decay-hence, the difference in the fields is not due to failure to achieve 'statistical equilibration'. The qualitative evolution of global average temperature is similar in all models after the initial adjustment period (i.e., for $t \geq 20 \tau$ ), with the globally averaged temperature exhibiting periodic fluctuations of amplitude $\sim 10-20 \mathrm{~K}$ on a timescale of 5-7 $\tau$ (due to the vortex life cycle discussed above) in all the models. The exception to this is the calculations with IGCM: in these calculations, temperature fluctuates with a clear 10-50 $\tau$ period, depending on the resolution, and the amplitude of the fluctuations is much larger $(\sim 40 \mathrm{~K})$ than in simulations with other cores. Note, the temperature fluctuations in simulations with CAM are also large at early times $(t \leq 20 \tau)$ but subsequently reduce, as discussed more in detail below.

Remarkably, the behavior of global average temperature in BOB and PEQMOD is nearly identical, up to the point when the equatorial symmetry is broken in PEQMOD (e.g., $t \sim 30 \tau$ in T21L20 resolution simulation). The clear periodicity present in the T21L20 and T42L20 BOB simulations disappears with increasing horizontal resolution (cf. red curve with green curve in top left panel of Fig. 9, for example). Note that, in all simulations, the global average temperature decreases by $10-20 \mathrm{~K}$ from the initial value of $1400 \mathrm{~K}$. The initial dip is related to the short timescale on which the large $T_{e}$ gradient is relaxed. For example, if the relaxation time is increased by $1 \tau$ everywhere in a BOB core simulation, the global average temperature decreases by less than $1 \mathrm{~K}$ (not shown). The adjustment demanded by the fast relaxation produces violent flows. Such a representation of thermal forcing is not physical—certainly its resulting flow is difficult to model accurately in current GCMs. Nevertheless, since we are primarily concerned with comparing the model cores, we use the representation here-for heuristic purposes.

Large amplitude fluctuations in the global average temperatures of IGCM and CAM simulations (particularly at early times in the latter) are associated with atmospheric thickness variations, caused by fluctuations in the surface pressure (recall that both cores use the $\sigma$-coordinate). This 'flapping' of the bottom boundary is absent in $p$-coordinate models with rigid top and bottom boundaries, in which surface pressure remains constant throughout the integration. By removing the bottom boundary away from the forcing region, we have found the atmospheric thickness variation to be greatly reduced in $\sigma$-coordinate models. Interestingly, as already mentioned, the fluctuations in the CAM core subside at $t \gtrsim 30 \tau$. This is likely due to the $\eta$-coordinate employed by the core.
Note that the flapping is not necessarily unphysical, and could be used to represent a physical phenomena at the 1 bar level if a hot extrasolar planet happens to have a natural, nonrigid boundary (e.g., jump in stratification, composition etc.) there. ${ }^{23}$ It is also possible to specify a free-surface boundary condition at the bottom in MITgcm. The specification replaces the condition, $\omega=0$ at $p=p_{\mathrm{r}}$, with $\omega=\mathrm{D} p_{s} / \mathrm{D} t$ at $p=p_{\mathrm{r}}$. With this boundary condition an additional prognostic equation for free surface pressure anomaly, called $\hat{\eta}$ in MITgcm (not to be confused with the $\eta$ associated with the CAM), is solved. However, given the strong forcing and violent flow that ensues, MITgcm in the default setting crashes with the boundary at 1 bar with the free-surface condition. This is due to the large undulation of the pressure surface, which can cause two or more pressure surfaces to intersect somewhere in the domain. Loss of single-valuedness such as this is not an issue in $\sigma$-coordinate model cores because surface pressure is not a coordinate surface.

It is clear from Fig. 9 that the cores are not numerically or visually converged. This is not surprising, especially for the pseudospectral cores, given that the superdissipation coefficient is decreased with increasing horizontal resolution. However, qualitative convergence in TC3 appears to be achieved at the lowest horizontal resolutions (i.e., T21, G32 and C16) in all the cores: at least, the qualitative behavior of flow and temperature appears to be the same at all resolutions. Given the behavior observed in TC2, however, we cannot completely rule out that this conclusion may need to be revised when simulations of substantially higher resolution (than those in this work) are carried out. In any case, we note here that the conclusion depends on the field or quantity considered, as we shall show later. For example, qualitative convergence is not achieved when the vorticity field is considered instead (Thrastarson and Cho, 2011).

In TC3, the total global energy is not conserved because $\dot{q} / c_{p} \neq 0$ in the thermodynamic equation. However, in the absence of momentum forcing, the global integral of absolute angular momentum is still conserved. While the angular momentum conservation has been almost exact in TC1 and TC2 (up to $\sim 0.02$ percent), in this test case the conservation property is strongly violated in some model cores-particularly in their default configurations. This is shown in Fig. 10, which presents time series of globally integrated absolute angular momentum (equation (17)). Each time series is normalized by its initial value of $1.77 \times 10^{32} \mathrm{~kg} \mathrm{~m}^{2} \mathrm{~s}^{-1}$.

As can be seen from Fig. 10, only BOB and PEQMOD conserve angular momentum exactly-and they do so at all resolutions (see top row of the figure). The conservation property of CAM and MITgcm in LL grid becomes better with increasing resolution. The periodic 'spinning' and 'de-spinning' of the atmosphere in IGCM by $\sim 10$ percent (see middle left panel of Fig. 10) is caused by variation in the atmospheric mass, due to large surface pressure variations. Clearly, angular momentum is poorly conserved in the calculations with the MITgcm in the

\footnotetext{
${ }^{23}$ Such a setup is common in Earth's middle atmosphere and climate studies to represent thermal and mechanical forcing.
} 

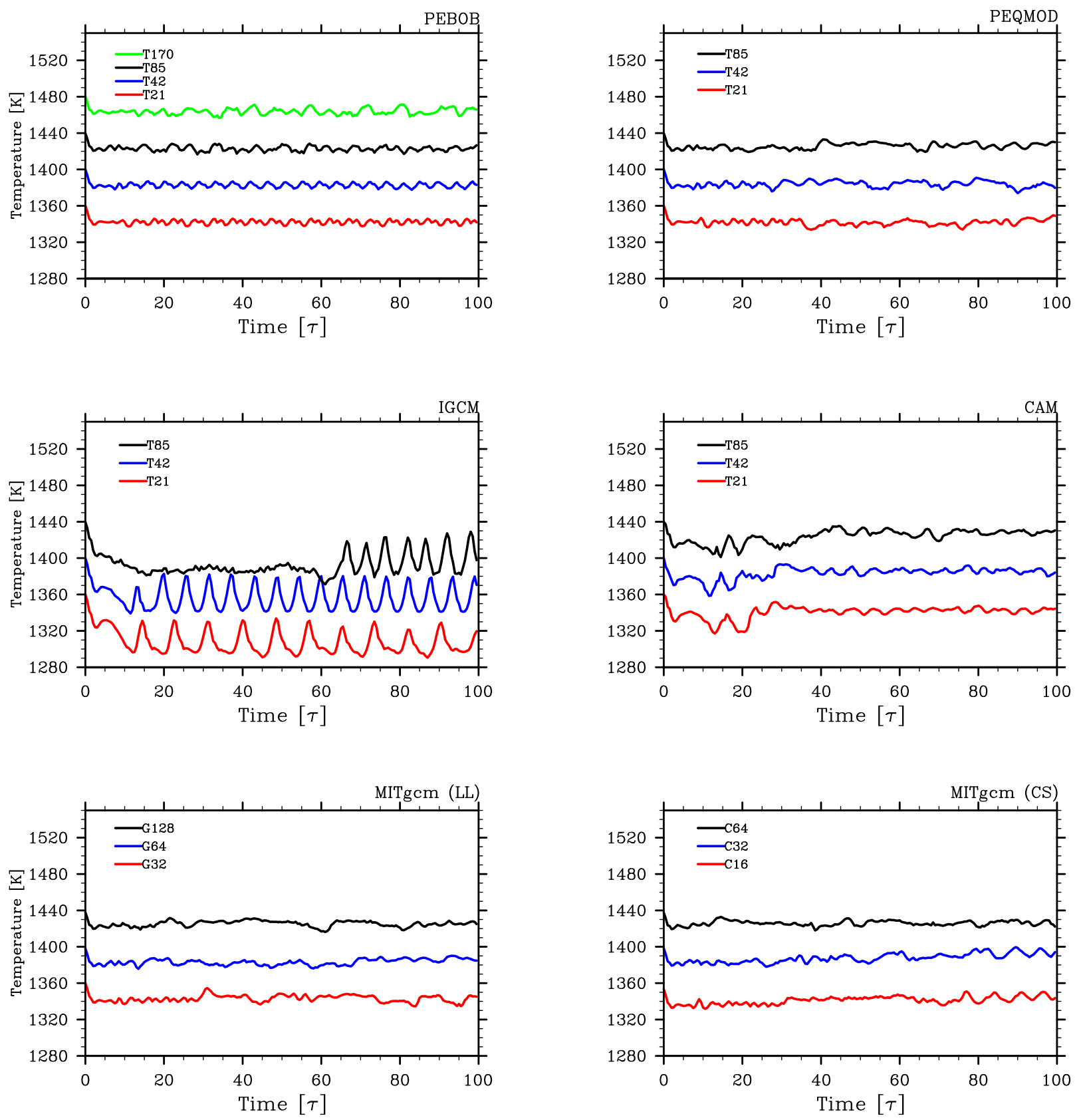

Figure 9: Time series of globally averaged temperature for the diabatic test case with different model cores at various horizontal resolutions. The top left panel is from simulations with BOB, the top right panel is from simulations with PEQMOD, the middle left panel is from simulations with IGCM, the middle right panel is from simulations with CAM, the bottom left panel is from simulations with MITgcm in LL grid and the bottom right panel is from simulations with MITgcm in CS grid. The curves have been offset from each other by $40 \mathrm{~K}$, with the temperature of the blue color having the correct scale. The panels show that all the calculations are equilibrated in temperature. They also show that qualitatively similar behavior in all the model calculations is not due to simple 'phasing' of the flow/temperature structures. For IGCM, there is a large, non-secular oscillatory behavior. 

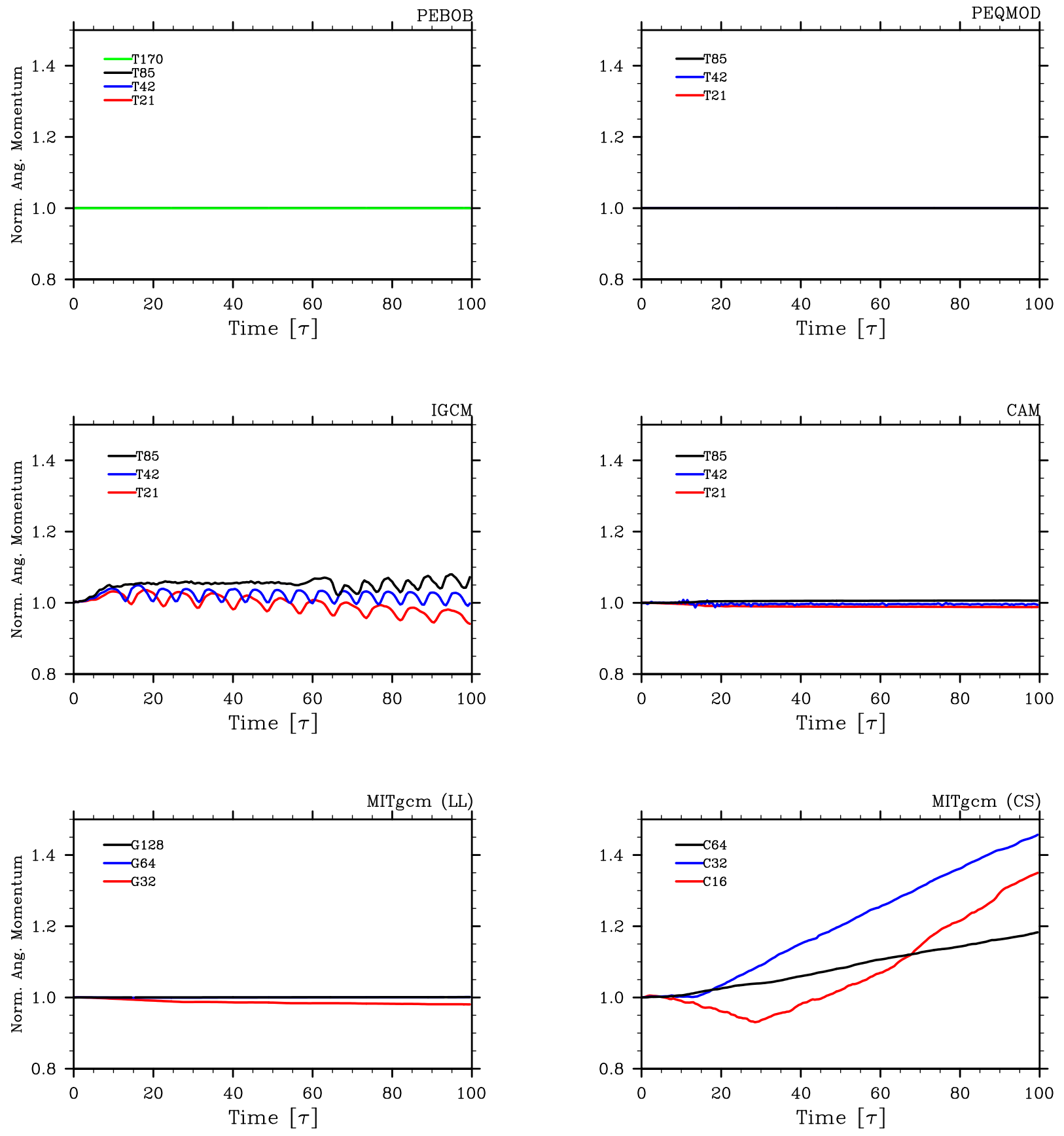

Figure 10: Time series of global angular momentum (normalized by initial value of $1.77 \times 10^{32} \mathrm{~kg} \mathrm{~m}^{2} \mathrm{~s}^{-1}$ ) for the diabatic test case with different model cores at various horizontal resolutions. The panel placements are as in Fig. 9 (i.e., BOB core at upper left, etc.). The total absolute angular momentum is exactly conserved at all resolutions in PEBOB and PEQMOD and at high resolutions in MITgcm in LL grid. It is somewhat poorly conserved in IGCM and not at all in MITgem in CS grid. 
CS grid, especially at C32 resolution: the total angular momentum increases by more than 45 percent at the end of the $100 \tau$ simulation. The conservation is actually better at the lower, C16 resolution, in terms of the time series at $t=100 \tau$. When these simulations are integrated for longer than $100 \tau$, the runaway angular momentum is associated with a zonally-averaged zonal flow which is strongly superrotating ${ }^{24}$ over a broad range of latitudes. For example, the transition to such a superrotating state occurs by $t \approx 200 \tau$ in C16 and C32 simulations.

The angular momentum runaway behavior in the MITgcm in CS grid simulations is caused by an instability associated with the Shapiro filter used in the simulations. When the strength of the filter is doubled (i.e., $\Delta t / \tau_{\text {shap }}=1 / 3$ ), angular momentum decreases over time leading to unphysical subrotation (i.e., westward flow of the atmosphere everywhere). This occurs by $t=100 \tau$. When the strength is halved (i.e., $\Delta t / \tau_{\text {shap }}=1 / 12$ ), not enough dissipation is supplied to the flow and the runaway still occurs and is more severe at an earlier time. Indeed, through an extensive study, we have found that a suitable strength of the Shapiro filter (which would conserve angular momentum exactly on the cubed sphere) does not exist for TC3. Note that this behavior is not just limited to the $\mathfrak{n}=2$ Shapiro filter. It occurs for higher power filters (e.g., $\mathfrak{n}=4$ and $\mathfrak{n}=6$ ) as well. Note also that, for this test case, the MITgcm in $\mathrm{CS}$ grid at $\mathrm{C} 16$ resolution (in the default setting) always crashes with $\mathfrak{n}=8$ Shapiro filter, independent of $\Delta t / \tau_{\text {shap }}$.

For completeness, we have also performed TC3 with the MITgcm core in both LL and CS grids (at G32 and C16 resolutions, respectively) with ordinary, Laplacian dissipation $\left(\nabla^{2}\right)$. The damping time in these simulations is chosen to be the same as in the simulations performed with the pseudospectral cores. The use of ordinary dissipation considerably improves the angular momentum conservation of MITgcm in CS grid (a monotonic increase of only $\sim 0.5$ percent at $t=100 \tau)$. However, there is now a significant ( $\sim 40$ percent) loss of angular momentum in the LL grid simulation using the same dissipation. In other words, the CS grid simulation is severely overdissipated compared to the LL grid simulation. From this we conclude that the dissipation is compensating the runaway in the CS grid. Therefore, the grid itself appears to be a significant source of the runaway behavior, with the Shapiro filter amplifying the behavior-at least in the model's default CS grid configuration.

As already discussed, the total energy with the applied forcing in TC3 is not expected to be conserved by any of the models. However, as shown below, the inclusion of Newtonian relaxation does not alter the total atmospheric energy budget by more than 5 percent. Fig. 11 shows a global integral of total energy as a function of time, normalized by the initial value of $2.2 \times 10^{28} \mathrm{~J}$. In all but the IGCM and CAM simulations the total energy steadily increases at the beginning by $\sim 2.5$ percent. Thereafter, BOB maintains the total energy at a nearly constant level (see top left panel of the figure). With the other cores, the total energy fluctuates by up to 5 percent, with largest fluctuations observed in IGCM simulations; at $t=100 \tau$ the

\footnotetext{
${ }^{24}$ i.e., $\bar{u}>\left(\Omega R_{\mathrm{p}} \sin ^{2} \phi / \cos \phi\right)$, where $\bar{u}=\bar{u}(\phi, s, t)$ is the zonally-averaged wind. Here, 'strongly' means $\bar{u}$ close to, or even exceeding, $3000 \mathrm{~m} \mathrm{~s}^{-1}$.
}

total energy for IGCM increases by 2-3 percent, on the average. The energy in the MITgcm simulations in CS grid also increases noticeably over time (particularly in the lower resolution simulations), consistent with the runaway angular momentum and transition to a superrotating state for $t \gtrsim 200 \tau$, discussed above. The total energy for CAM shows a slight increases, by $\sim 1$ percent, over the initial value, after settling from large initial fluctuations; but, PEQMOD and MITgcm LL grid simulations show the energy decrease slightly by $\sim 1.5$ percent, after the initial rise of $\sim 2.5$ percent mentioned above.

In the process of thoroughly verifying our results, we have discovered, as already noted, two additional advection schemes for the vector invariant momentum equation for the MITgcm CS grid. Of the two undocumented schemes, one explicitly conserves energy (Sadourny, 1975) and the other conserves energy and enstrophy (Burridge and Haseler, 1977). While detailed discussion on the behaviours of MITgcm with these two 'non-default' schemes are reported elsewhere (Polichtchouk and Cho, in prep.), we note that the angular momentum conservation is noticeably improved in TC3, particularly with the energy conserving scheme. For example, with the use of the energy conserving scheme the angular momentum in TC3 decreases by 33 percent at $\mathrm{C} 16$ resolution, by 6 percent at $\mathrm{C} 32$ resolution and by 2 percent at $\mathrm{C} 64$ resolution at the end of the $100 \tau$ simulation. Because these decreases are monotonic with time longer time integrations would result in a more significant violation of angular momentum consevation in TC3. We also note that the two undocumented schemes do not improve the performance in TC1 and TC2, compared to the default enstrophy conserving scheme.

As a closing remark, somewhat disconcertingly, we have noticed that the model cores are perceptibly sensitive to small, perhaps 'uncontrollable', changes in input parameters when subject to the strong forcing, as in TC3. We have already shown that the simulation results are different 1 ) between different model cores at the same or comparable resolution and 2) between different resolutions with the same model core. However, the simulation results can also differ with the same core at the same resolution. For example, simulations with PEQMOD at T42L20 resolution with single, double and quadruple precision produce quantitatively unconverged results (although they may be qualitatively converged). In addition to the onset of the north-south symmetry breaking, we have found a noticeable phase difference between the solutions. Such phase differences are significant for predicting precise flow and temperature patterns. Moreover, we have found the phase differences to occur in a model core with identical setup and precision when the core was simply compiled with a different compiler or the same compiler on a different computing platform. It is important to note that the above issues did not play significant roles in TC1 and TC2 because 'violent' (i.e., strongly unbalanced) flows are not involved. Given this, we do not expect the results presented in TC3 to be exactly reproducible when a different compiler is used and/or on different platforms: slight phase variations are nominally expected. 

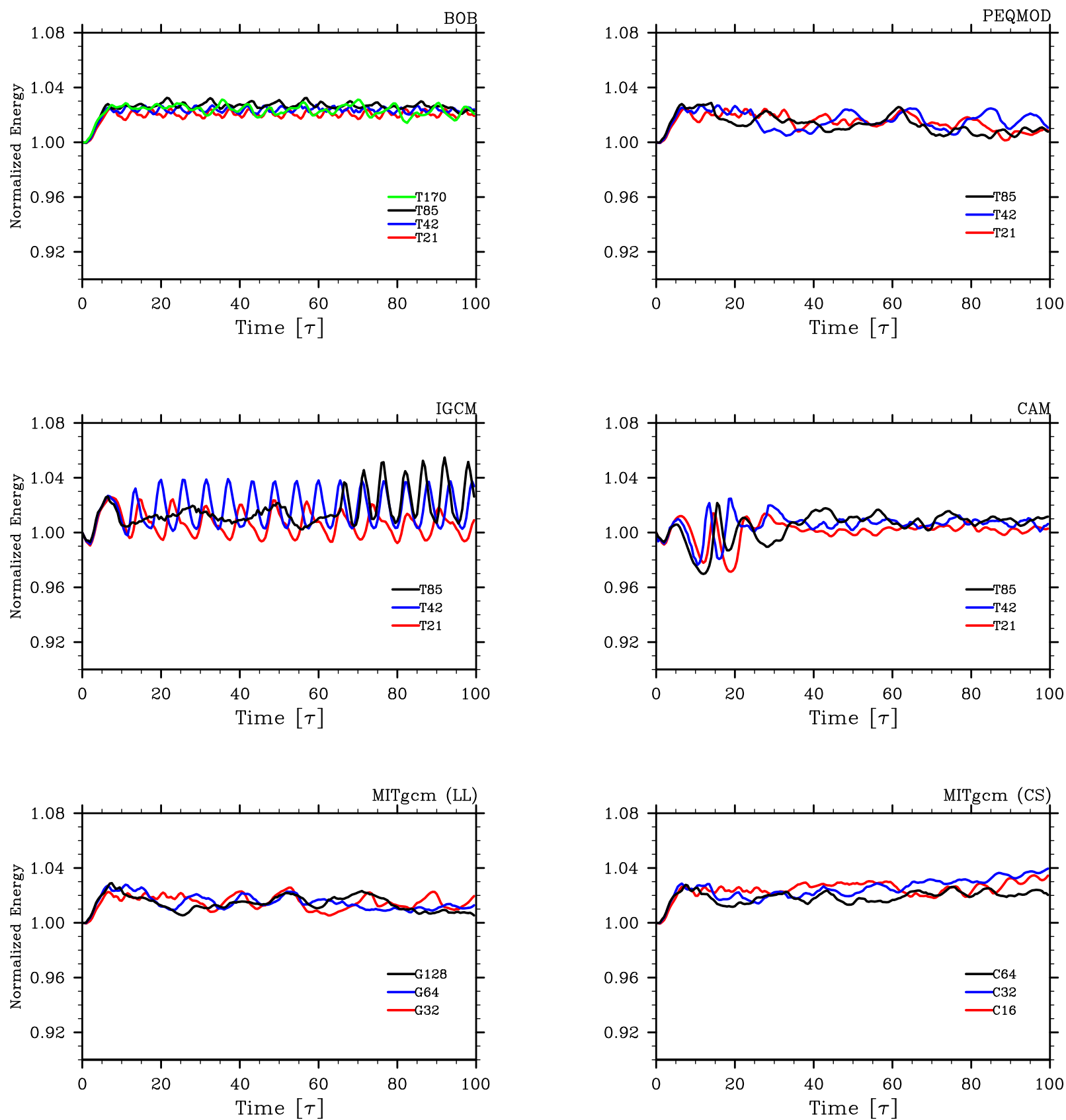

Figure 11: Time series of global total energy (normalized by initial value of $2.2 \times 10^{28} \mathrm{~J}$ ) for test case 3 with different model cores at varying horizontal resolutions. The panel placements are as in Fig. 9 (i.e. BOB core at upper left, etc.). All the simulations appear to be well or roughly equilibrated, with the possible exception of the lower resolution simulations with the MITgcm core in CS grid. The total energy fluctuations are correlated with the temperature fluctuations (cf. Fig. 9). 


\section{Summary and Discussion}

Intercomparison and benchmarking of GCMs, and in particular their cores, are necessary for assessing the efficacy of models and for understanding the physical properties of atmospheres. While such testing is common practice in Earth and some Solar System planet studies, only three tests (e.g., Rauscher and Menou, 2010; Heng et al., 2011; Bending et al., 2013) have been attempted for hot extrasolar planet GCMs. Here, we subject five GCMs currently used in the hot extrasolar planet studies to three benchmark tests. The tested GCMs are: BOB, PEQMOD, IGCM, CAM, and MITgcm. These models employ a range of numerical algorithms for the spatial discretization and explicit viscosity: respectively, pseudospectral with pseudospectral filtering (BOB, PEQMOD, IGCM, and CAM) and finite volume with differenced, pole (zonal), and Shapiro filtering (MITgcm). All the GCMs solve the dry, hydrostatic primitive equations and are subjected to identical tests. From least to most stringent, these tests are: 1) the steady-state test case (TC1); 2) the baroclinic wave test case (TC2); and, 3) the diabatic test case (TC3). In all three test cases, all of the models are tested at varying horizontal resolutions to assess numerical convergence. Both inter- and intra-comparisons are carried out.

TC1 assesses how a model is able to maintain a balanced initial condition in the form of a midlatitude eastward jet with no applied dissipation, before gravity waves and numerical noise degrade the jet. With the exception of MITgcm employing the CS grid, all models maintain the true, steady-state solution very well throughout the time of integration $(t=20 \tau)$. The special corners in the CS mesh quickly degrade the balanced state; and, in the absence of any dissipation, the imbalance causes the model simulations to crash at early time when using the default advection scheme $\mathrm{e}^{25}$ : for example, the simulation at $\mathrm{C} 64$ resolution crashes at $t=4.5 \tau$. Of all the models, BOB and PEQMOD maintain the true solution the best (see, e.g., Fig. 3).

In TC2, a temperature perturbation applied to the steady-state initial condition of TC1 triggers a nonlinear evolution of a baroclinic wave. The emergence of sharp fronts from baroclinic instability necessitates the use of flow viscosity, unlike in TC1. We have chosen ordinary Laplacian (i.e., $\nabla^{2}$ ) dissipation. While all model calculations permit baroclinic instability, only pseudospectral ones appear to be visually converged, and this occurs at a horizontal resolution of T85 (corresponding to 85 total and zonal modes each in the spherical harmonic expansion-a resolution above most current extrasolar planet simulations). Solutions with MITgcm are not converged at the highest resolutions investigated here (i.e., G128 and C64). While the most unstable mode of the unstable jet is approximately 3-4 (see Fig. 4 and Fig. 5), the presence of the special corner points on the cubedsphere grid produces an unstable wave field with mode greater than 3-4 (see Fig. 5 bottom right panel). This behavior is significant for model predictions and observational confirmations.

\footnotetext{
${ }^{25}$ Note that the steady-state simulations with energy and energy and enstrophy conserving schemes do not crash before $t=20 \tau$. However, the physical space fields are inundated with grid-scale noise at early time and the simulations are clearly unbalanced.
}

While the first two test cases are adiabatic, the third case is diabatic. For some regions of the planet atmosphere, diabatic forcing is necessary. In this work, as in most hot extrasolar planet studies thus far, we apply a simple Newtonian relaxation parameterization to represent the heating and cooling in the modeled atmosphere. Here, the atmosphere is 'spun-up' from an initial isothermal condition at rest. Note that, although many past studies have employed a similar setup, there is no general agreement on the robustness of the flow and temperature distributions produced by the simulations. By employing an identical setup in all the models, the aim of TC3 has been to clearly assess whether (and how much) the non-robustness of the results is intrinsic to the numerical model employed. We emphasize again that without such tests, the simulation communityindeed the extrasolar planet community as a whole-would not have a baseline for any consensus. For this reason, we employ a biharmonic $\left(\nabla^{4}\right)$ superviscosity in pseudospectral models and a power-two $(\mathfrak{n}=2)$ Shapiro filter in MITgcm so that our comparisons are equatable among the models tested in this study and shed some light on the results of past studies. ${ }^{26}$

Unlike in the first two test cases, the extreme forcing condition of the third test case produces a range of behaviors in the model calculations. While there are some qualitatively similar features (e.g., a time-variable quadrupole flow structure), the location and magnitude of the hottest and coldest regions are not same in the model calculations. In large part, this is due to significant phase differences in the computed fields. Moreover, apart from BOB and IGCM, all models break the flow symmetry about the equator relatively early on, before $t=100 \tau$ (see Fig. 8). This behavior may be somewhat surprising as the forcing is north-south symmetric and no initial noise is present to break the symmetry, but machine precision or coding inexactitude eventually break the symmetry in all the cores tested.

Throughout this work we have paid careful attention to the conservation properties of the numerical models. In TC1 and TC2, the angular momentum and total energy conservations are well fulfilled by all the models. In TC3, however, we have found that - apart from BOB and PEQMOD — no other model conserves angular momentum exactly. The MITgcm in CS grid with Shapiro filter perform the poorest in this case, with the total absolute angular momentum increasing by as much as 45 percent at the end of the integration $(t=100 \tau)$. As pointed out in Thrastarson and Cho (2011) and Polichtchouk and Cho (2012), the normally 'relatively harmless' small nonconservation in Earth-like conditions, for which the GCMs have been constructed and tested, could be exacerbated in the hot extrasolar planet condition. This is because of the exacting requirements the condition places on the numerics. Such model behaviors should be carefully taken into account, when performing hot extrasolar planet simulations. We emphasize this point in this work because we note that runaway (or decaying) angular momentum is not necessarily apparent from looking at the flow pattern alone (see Fig. 8 bottom row). Long-time inte-

\footnotetext{
${ }^{26}$ We remind the reader here that the MITgcm in this default CS grid configuration possesses the least amount of angular momentum runaway at $t=100 \tau$ with $\mathfrak{n}=2$.
} 
gration of such a simulation leads to an atmosphere that superrotates in a manner similar to those reported in previous studies.

GCMs are complex. Getting them to run properly and verifying their results is not trivial. Trade-offs between accuracy, speed and algorithmic/coding simplicity are always made; and, even when such things are well-understood theoretically, the actual behavior of the model is not always 'stable' or uniform across problems. This then also raises the complexity of interpreting the results. In this work, we have endeavoured to fairly assess the performance of a number of GCMs - covering a good cross-section of algorithms, grids and treatment of explicit viscosity. For the most part, the tested models behave well and similarly to each other, but with some unexpected results. Although we have extensively checked our calculations here and despite our best effort to provide all the details of the calculations, it is still possible-especially given some of the findings presented in TC3 - that other studies may obtain different results. Broadly, we offer this work for other studies to reproduce and to build on, as it seems clear to us that that is necessary for advancement in theory and modelling of hot extrasolar planet atmospheres.

\section{Acknowledgments}

The authors thank three anonymous reviewers for helpful comments, which improved this manuscript. This work has been supported by the Science and Technology Facilities Council (STFC) research studentships to I.P. and C.W., STFC grant PP/E001858/1 and Westfield Small Grant (WSG) to J.Y-K.C. H.T.T was supported by an appointment to the NASA Postdoctoral Program at the Jet Propulsion Laboratory, administered by Oak Ridge Associated Universities through a contract with NASA. H.T.T. was also supported in part by the National Science Foundation under Grant No. PHYS-1066293 and the hospitality of the Aspen Center for Physics. O.M.U. and M.T.J. acknowledge support from STFC and WSG, respectively.

\section{References}

Adcroft A., et al., 2012, 'MITgcm User Manual'

Anderson J. L., et al., 2004, The new GFDL global atmosphere and land model AM2-LM2: Evaluation with prescribed SST simulations, J. Cli., 17, 46414673

Arakawa A., Lamb V., 1977, Computational design and the basic dynamical processes of the UCLA general circulation model, Methods Comput. Phys., $17,173-265$

Arakawa A., Moorthi S., 1988, Baroclinic instability in vertically discrete systems, J. Atmos. Sci., 45, 1688-1707

Asselin R., 1972, Frequency filter for time integrations, Mon. Wea. Rev., 100, 487-490

Beaulieu J-P., et al., 2011, Methane in the atmosphere of the transiting hot Neptune GJ436b?, ApJ, 731, 16-28

Bending V. L., Lewis S. R., Kolb U., 2013, Benchmark experiments with global climate models applicable to extrasolar gas giant planets in the shallow atmosphere approximation, MNRAS, 428, 2874-2884

Blackburn M., 1985, 'Program Description for the Multi-level Global Spectral Model'

Blackburn M., et al., 2013, The Aqua Planet Experiment (APE): Control SST simulation, J. Met. Soc. Japan, 91A

Boer G. L., Denis B., 1997, Numerical convergence of the dynamics of a GCM, Cli. dyn., 13, 359-374
Boyd, J. P. 2000, Chebyshev and Fourier Spectral Methods (2nd ed.; New York: Dover)

Burridge, D. M., Haseler, J., 1977, A model for medium range weather forecast - adiabatic formulation, ECMWF Tech. Rep., No.4

Canuto C., Hussaini M. Y., Qarteroni A., Zang T. A., 1988, Spectral Methods in Fluid Dynamics (New York: Springer)

Cho J. Y-K., 2008, Atmospheric dynamics of tidally synchronized extrasolar planets, Phil. Trans. R. Soc. A, 366, 4477-4488

Cho J. Y-K., Polvani L., 1996, The emergence of jets and vortices in freely evolving, shallow-water turbulence on a sphere, Phys. Fluids, 8, 1531-1552

Cho J. Y-K., Menou K., Hansen B. M. S., Seager S., 2008, Atmospheric circulation of close-in extrasolar giant planets. I. global, barotropic, adiabatic simulations, ApJ, 675, 817-845

Collins W. D.,et al., 2004, 'Description of the NCAR Community Atmosphere Model (CAM 3.0)'

Cooper C. S., Showman A. P., 2005, Dynamics and disequilibrium carbon chemistry in hot Jupiter atmospheres, with application to HD 209458b, ApJ, 629, L45-L48

Durran D. R., 1999, 'Numerical Methods for Wave Equations in Geophysical Fluid Dynamics' (Springer, New York)

Eliassen E., Machenhauer B., Rasmussen E., 1970, Copenhagen Univ., Inst. Teoretisk Meteorologi, Tech. Rep. 2

Gill A. E., 1980, Some simple solutions for for heat-induced tropical circulation, Q. J. Roy. Met. Soc., 106, 447-462

Held I. M., Suarez M. J., 1994, A proposal for the intercomparison of the dynamical cores of atmospheric general circulation models, Bull. A.M.S., 75, $1825-1830$

Heng K., Menou K., Phillips P. J., 2011, Atmospheric circulation of tidallylocked exoplanets: a suite of benchmark tests for dynamical solvers, $\mathrm{MN}$ RAS, 413, 2380-2402

Holton J. R, 1992 'Introduction to Dynamic Meteorology' (Academic Press, San Diego)

Jablonowski C., Williamson D. L., 2006, A baroclinic instability test case for atmospheric model dynamical cores, Q. J. Roy. Met. Soc., 132, 2943-2975

Kasahara A., 1974, Various vertical coordinate systems used for numerical weather prediction, Mon. Wea. Rev., 102, 509-522

Kaspi Y., Flierl, G. Showman, A. P., 2009, The deep wind structure of the giant planets: Results from an anelastic general circulation model, Icarus, 202, 525-542

Lauritzen P. H., Jablonowski C., Taylor M. A., Nair R. D., 2010, Rotated versions of the Jablonowski steady-state and baroclinic wave test cases: A dynamical core intercomparison, J. Adv. Model. Earth Syst., 2, 1-41

Lauritzen P. H., Jablonowski C., Taylor M. A., Nair R. D., 2011, 'Numerical Techniques for Global Atmospheric Models' (Springer, New York)

Lebonnois S., et al., 2013, Models of Venus atmosphere, 'Towards Understanding the Climate of Venus: Application of Terrestrial Models to Our Sister Planet' (Springer, New York), 129-156

Lewis N. K., Showman A. P., Fortney J. J., Marley M. S., Freedman R. S., Lodders K., 2010, Atmospheric circulation of eccentric hot Neptune GJ436b, ApJ, 720, 344-356

Lorenz E. N., 1960, Energy and numerical weather prediction, Tellus, 12, 364 373

Matsuno T., 1966, Quasi-geostrophic motions in the equatorial area, J. Met. Soc. Japan, 44, 25-43

Menou K., Rauscher E., Atmospheric Circulation of Hot Jupiters: A Shallow Three-Dimensional Model, 2009, ApJ 700, 887-897

Orszag A., 1970, Transform method for calculation of vector-coupled sumsApplication to spectral form of vorticity equation, J. Atmos. Sci., 27, 890-895

Polichtchouk, I., Cho Y-K. J., 2012, Baroclinic instability on hot extrasolar planets, MNRAS, 424, 1307-1326

Polvani L. M., Scott R. K., Thomas S. J., 2004, Numerically Converged Solutions of the Global primitive equations for testing the dynamical core of atmospheric GCMs, Mon. Wea. Rev., 132, 2539-2552

Rauscher E., Menou K., 2010, Three dimensional modeling of hot Jupiter atmospheric flows, ApJ, 714, 1334-1342

Robert A., 1966, The integration of a low order spectral form of the primitive meteorological equations, J. Met. Soc. Japan, 44, 237-245

Sadourny R., 1975, The dynamics of finite-difference models of the shallow water equations, JAS, 32, 680-689

Saravanan R., 1992, 'A Mechanistic Spectral Primitive Equation Model using Pressure Coordinates'. 
Scott R. K., Rivier L., Loft R., Polvani L. M., 2003, NCAR Technical Note

Shapiro R., 1970, Smoothing, filtering and boundary effects, Review of Geophysics and Space Physics, 8(2), 359-387

Showman A. P., Cooper C. S., Fortney J. J., Marley M. S., 2008, Atmospheric Circulation of Hot Jupiters: Three-dimensional circulation models of HD 209458b and HD 189733b with simplified forcing, ApJ, 682, 559576

Showman A. P., Cho J. Y-K., Menou K., 2010, in EXOPLANETS, ed., S. Seager, Tuscon: University of Arizona Press

Showman A. P., Fortney J. J., Lian Y., Marley M. S., Freedman R. S., Knutson H. A., Charbonneau D., 2009, Atmospheric circulation of hot Jupiters: Coupled radiative-dynamical general circulation model simulations of HD 189733b and HD 209458b, ApJ, 699, 564-584

Simmons A. J., Burridge M. M., 1981, An energy and angular-momentum conserving vertical finite- difference scheme and hybrid vertical coordinates, Mon. Wea. Rev., 109, 758-766

Simmons A. J., Hoskins B. J, 1979, The downstream and upstream development of unstable baroclinic waves, J. Atmos. Sci., 36, 1239-1259

Suarez M. J., Takacs L. L. 1995, Technical report series on global modeling and data assimilation. Vol.5: Documentation of the AIRES/GEOS dynamical core, v.2

Thorncroft C. D., Hoskins B. J., McIntyre M. E., 1993, Two paradigms of baroclinic-wave life-cycle behaviour, Q. J. R. Meteorol. Soc., 119, 17-55

Thrastarson H. Th., Cho J. Y-K., 2010, Effects of initial flow on close-in planet atmospheric circulation, ApJ, 716, 144-153

Thrastarson H. Th., Cho J. Y-K., 2011, Relaxation time and dissipation interaction in hot planet atmospheric flow simulations, ApJ, 729, 117-128

\section{Appendix A. Tables of Values for Test Cases}


Table A.1: Table of vertical and horizontal grid resolutions as well as other parameters needed for reproduction of steady-state case with pseudospectral cores. Note, PEQMOD has an additional equatorial latitude point (i.e. T21 pseudospectral resolution corresponds to $33 \times 64$ grid points, T42 to $65 \times 128$ grid points etc.). BOB only has been integrated at resolution T170L20.

\begin{tabular}{cccccc}
\hline $\begin{array}{c}\text { Horizontal } \\
\text { Resolution }\end{array}$ & $\begin{array}{c}\text { Vertical } \\
\text { Resolution }\end{array}$ & $\begin{array}{c}\text { Gaussian Grid } \\
(\text { lon } \times \text { lat })\end{array}$ & $\begin{array}{c}\text { Timestep } \\
(\Delta t)[\mathrm{s}]\end{array}$ & Hyperdissipation & $\begin{array}{c}\text { Robert-Asselin } \\
\text { Coefficient }(\epsilon)\end{array}$ \\
\hline T21 & L20 & $64 \times 32$ & 120 & No & 0.001 \\
T42 & L20 & $128 \times 64$ & 60 & No & 0.001 \\
T85 & L20 & $256 \times 128$ & 30 & No & 0.001 \\
T170 & L20 & $512 \times 256$ & 15 & No & 0.001 \\
\hline
\end{tabular}

Table A.2: As in Table A.1, but for MITgcm in longitude-latitude (LL) grid. Note, the computational grid size below should not be compared directly with the Gaussian grid size in Table A.1, as the pseudospectral evolves the fields in spectral space: the Gaussian grid is used only to evaluate the nonlinear products and de-alias the fields.

\begin{tabular}{ccccccc}
\hline $\begin{array}{c}\text { Horizontal } \\
\text { Resolution }\end{array}$ & $\begin{array}{c}\text { Vertical } \\
\text { Resolution }\end{array}$ & $\begin{array}{c}\text { Computational Grid } \\
(\text { lon } \times \text { lat })\end{array}$ & $\begin{array}{c}\text { Timestep } \\
(\Delta t)[\mathrm{s}]\end{array}$ & $\begin{array}{c}\text { Harmonic } \\
\text { Dissipation }\end{array}$ & $\begin{array}{c}\text { Zonal (FFT) } \\
\text { Filter }\end{array}$ & $\begin{array}{c}\text { Shapiro } \\
\text { Filter }\end{array}$ \\
\hline G32 & L20 & $64 \times 32$ & 120 & No & Yes & No \\
G64 & L20 & $128 \times 64$ & 60 & No & Yes & No \\
G128 & L20 & $256 \times 128$ & 30 & No & Yes & No \\
\hline
\end{tabular}

Table A.3: Same as Table A.1, but for MITgcm in cubed-sphere (CS) grid. N.B., the C16 and C64 CS grids have been generated by us with MATLAB routines provided by MITgcm support; but, the MATLAB generated C32 grid comes included with the MITgcm.

\begin{tabular}{ccccccc}
\hline $\begin{array}{c}\text { Horizontal } \\
\text { Resolution }\end{array}$ & $\begin{array}{c}\text { Vertical } \\
\text { Resolution }\end{array}$ & $\begin{array}{c}\text { Computational Grid } \\
\text { (irregular) }\end{array}$ & $\begin{array}{c}\text { Timestep } \\
(\Delta t)[\mathrm{s}]\end{array}$ & $\begin{array}{c}\text { Harmonic } \\
\text { Dissipation }\end{array}$ & $\begin{array}{c}\text { Zonal (FFT) } \\
\text { Filter }\end{array}$ & $\begin{array}{c}\text { Shapiro } \\
\text { Filter }\end{array}$ \\
\hline C16 & L20 & $6 \times 16 \times 16$ & 120 & No & No & No \\
C32 & L20 & $6 \times 32 \times 32$ & 60 & No & No & No \\
C64 & L20 & $6 \times 64 \times 64$ & 30 & No & No & No \\
\hline
\end{tabular}

Table A.4: Table of vertical and horizontal grid resolutions as well as other parameters needed for reproduction of baroclinic wave test case with pseudospectral cores. Note, as above, PEQMOD has an additional equatorial latitude point (i.e. T21 pseudospectral resolution corresponds to $33 \times 64$ grid points, T42 to $65 \times 128$ grid points etc.). Also, note, only BOB has been integrated at resolution T170L20.

\begin{tabular}{cccccccc}
\hline $\begin{array}{c}\text { Horizontal } \\
\text { Resolution }\end{array}$ & $\begin{array}{c}\text { Vertical } \\
\text { Resolution }\end{array}$ & $\begin{array}{c}\text { Guassian Grid } \\
(\text { lon } \times \text { lat })\end{array}$ & $\begin{array}{c}\text { Timestep } \\
(\Delta t)[\mathrm{s}]\end{array}$ & Hyperdissipation & $\begin{array}{c}\text { Dissipation } \\
\text { Order }(\mathfrak{p})\end{array}$ & $\begin{array}{c}\text { Dissipation Coefficient } \\
\left(v_{2}\right)\left[\mathrm{m}^{2} \mathrm{~s}^{-1}\right]\end{array}$ & $\begin{array}{c}\text { Robert-Asselin } \\
\text { Coefficient }(\epsilon)\end{array}$ \\
\hline T21 & L20 & $64 \times 32$ & 120 & Yes & 1 & $2 \times 10^{7}$ & 0.001 \\
T42 & L20 & $128 \times 64$ & 60 & Yes & 1 & $2 \times 10^{7}$ & 0.001 \\
T85 & L20 & $256 \times 128$ & 30 & Yes & 1 & $2 \times 10^{7}$ & 0.001 \\
T170 & L20 & $512 \times 256$ & 15 & Yes & 1 & $2 \times 10^{7}$ \\
\hline
\end{tabular}

Table A.5: Same as Table A.4, but for MITgem in LL grid.

\begin{tabular}{cccccccc}
\hline $\begin{array}{c}\text { Horizontal } \\
\text { Resolution }\end{array}$ & $\begin{array}{c}\text { Vertical } \\
\text { Resolution }\end{array}$ & $\begin{array}{c}\text { Computational Grid } \\
(\text { lon } \times \text { lat })\end{array}$ & $\begin{array}{c}\text { Timestep } \\
(\Delta t)[\mathrm{s}]\end{array}$ & $\begin{array}{c}\text { Harmonic } \\
\text { Dissipation }\end{array}$ & $\begin{array}{c}\text { Dissipation Coefficient } \\
\left(v_{2}\right)\left[\mathrm{m}^{2} \mathrm{~s}^{-1}\right]\end{array}$ & $\begin{array}{c}\text { Zonal (FFT) } \\
\text { Filter }\end{array}$ & $\begin{array}{c}\text { Shapiro } \\
\text { Filter }\end{array}$ \\
\hline G32 & L20 & $64 \times 32$ & 120 & Yes & $2 \times 10^{7}$ & Yes & No \\
G64 & L20 & $128 \times 64$ & 60 & Yes & $2 \times 10^{7}$ & Yes & No \\
G128 & L20 & $256 \times 128$ & 30 & Yes & $2 \times 10^{7}$ & Yes & No \\
\hline
\end{tabular}


Table A.6: Same as Table A.4 but for MITgcm in CS grid.

\begin{tabular}{cccccccc}
\hline $\begin{array}{c}\text { Horizontal } \\
\text { Resolution }\end{array}$ & $\begin{array}{c}\text { Vertical } \\
\text { Resolution }\end{array}$ & $\begin{array}{c}\text { Computational Grid } \\
\text { (irregular) }\end{array}$ & $\begin{array}{c}\text { Timestep } \\
(\Delta t)[\mathrm{s}]\end{array}$ & $\begin{array}{c}\text { Harmonic } \\
\text { Dissipation }\end{array}$ & $\begin{array}{c}\text { Dissipation Coefficient } \\
\left(v_{2}\right)\left[\mathrm{m}^{2} \mathrm{~s}^{-1}\right]\end{array}$ & $\begin{array}{c}\text { Zonal (FFT) } \\
\text { Filter }\end{array}$ & $\begin{array}{c}\text { Shapiro } \\
\text { Filter }\end{array}$ \\
\hline C16 & L20 & $6 \times 16 \times 16$ & 120 & Yes & $2 \times 10^{7}$ & No & No \\
C32 & L20 & $6 \times 32 \times 32$ & 60 & Yes & $2 \times 10^{7}$ & No & No \\
C64 & L20 & $6 \times 64 \times 64$ & 30 & Yes & $2 \times 10^{7}$ & No & No \\
\hline
\end{tabular}

Table A.7: Table of vertical and horizontal grid resolutions as well as other parameters needed for reproduction of diabatic forcing test case with pseudospectral cores. Note, as above, PEQMOD has an additional equatorial latitude point (i.e. T21 pseudospectral resolution corresponds to $33 \times 64$ grid points, T42 to $65 \times 128$ grid points etc.). Here also, only BOB has been integrated at resolution T170L20.

\begin{tabular}{cccccccc}
\hline $\begin{array}{c}\text { Horizontal } \\
\text { Resolution }\end{array}$ & $\begin{array}{c}\text { Vertical } \\
\text { Resolution }\end{array}$ & $\begin{array}{c}\text { Gaussian Grid } \\
(\text { lon } \times \text { lat })\end{array}$ & $\begin{array}{c}\text { Timestep } \\
(\Delta t)[\mathrm{s}]\end{array}$ & Hyperdissipation & $\begin{array}{c}\text { Dissipation } \\
\text { Order }(\mathfrak{p})\end{array}$ & $\begin{array}{c}\text { Dissipation Coefficient } \\
v_{4}\left[\mathrm{~m}^{4} \mathrm{~s}^{-1}\right]\end{array}$ & $\begin{array}{c}\text { Robert-Asselin } \\
\text { Coefficient }(\epsilon)\end{array}$ \\
\hline T21 & L20 & $64 \times 32$ & 240 & Yes & 2 & $1 \times 10^{23}$ \\
T42 & L20 & $128 \times 64$ & 120 & Yes & 2 & $5 \times 10^{22}$ & 0.01 \\
T85 & L20 & $256 \times 128$ & 60 & Yes & 2 & $1 \times 10^{22}$ \\
T170 & L20 & $512 \times 256$ & 30 & Yes & 2 & $5 \times 10^{21}$ \\
\hline
\end{tabular}

Table A.8: Same as Table A.7 but for MITgcm in LL grid.

\begin{tabular}{ccccccccc}
\hline $\begin{array}{c}\text { Horizontal } \\
\text { Resolution }\end{array}$ & $\begin{array}{c}\text { Vertical } \\
\text { Resolution }\end{array}$ & $\begin{array}{c}\text { Computational Grid } \\
(\text { lon } \times \text { lat })\end{array}$ & $\begin{array}{c}\text { Timestep } \\
(\Delta t)[\mathrm{s}]\end{array}$ & $\begin{array}{c}\text { Harmonic } \\
\text { Dissipation }\end{array}$ & $\begin{array}{c}\text { Zonal (FFT) } \\
\text { Filter }\end{array}$ & $\begin{array}{c}\text { Shapiro } \\
\text { Filter }\end{array}$ & $\begin{array}{c}\text { Filter } \\
\text { Power }(\mathfrak{n})\end{array}$ & $\begin{array}{c}\text { Filter Parameter } \\
\left(\tau_{\text {shap }}\right)[\mathrm{s}]\end{array}$ \\
\hline G32 & L20 & $64 \times 32$ & 240 & No & Yes & Yes & 2 & 1440 \\
G64 & L20 & $128 \times 64$ & 120 & No & Yes & Yes & 2 & 720 \\
G128 & L20 & $256 \times 128$ & 60 & No & Yes & Yes & 2 & 360 \\
\hline
\end{tabular}

Table A.9: Same as Table A.7 but for MITgcm in CS grid.

\begin{tabular}{ccccccccc}
\hline $\begin{array}{c}\text { Horizontal } \\
\text { Resolution }\end{array}$ & $\begin{array}{c}\text { Vertical } \\
\text { Resolution }\end{array}$ & $\begin{array}{c}\text { Computational Grid } \\
\text { (irregular) }\end{array}$ & $\begin{array}{c}\text { Timestep } \\
(\Delta t)[\mathrm{s}]\end{array}$ & $\begin{array}{c}\text { Harmonic } \\
\text { Dissipation }\end{array}$ & $\begin{array}{c}\text { Zonal (FFT) } \\
\text { Filter }\end{array}$ & $\begin{array}{c}\text { Shapiro } \\
\text { Filter }\end{array}$ & $\begin{array}{c}\text { Filter } \\
\text { Power }(\mathfrak{n})\end{array}$ & $\begin{array}{c}\text { Filter Parameter } \\
\left(\tau_{\text {shap }}\right)[\mathrm{s}]\end{array}$ \\
\hline C16 & L20 & $6 \times 16 \times 16$ & 240 & No & No & Yes & 2 & 1440 \\
C32 & L20 & $6 \times 32 \times 32$ & 120 & No & No & Yes & 2 & 720 \\
C64 & L20 & $6 \times 64 \times 64$ & 60 & No & No & Yes & 2 & 360 \\
\hline
\end{tabular}

